ESAIM: M2AN 46 (2012) 1175-1199

DOI: $10.1051 / \mathrm{m} 2 \mathrm{an} / 2011073$
ESAIM: Mathematical Modelling and Numerical Analysis

www.esaim-m2an.org

\title{
ROBUST DOMAIN DECOMPOSITION PRECONDITIONERS FOR ABSTRACT SYMMETRIC POSITIVE DEFINITE BILINEAR FORMS
}

\author{
Yalchin Efendiev ${ }^{1}$, Juan Galvis ${ }^{1}$, Raytcho Lazarov ${ }^{1}$ and Joerg Willems ${ }^{2}$
}

\begin{abstract}
An abstract framework for constructing stable decompositions of the spaces corresponding to general symmetric positive definite problems into "local" subspaces and a global "coarse" space is developed. Particular applications of this abstract framework include practically important problems in porous media applications such as: the scalar elliptic (pressure) equation and the stream function formulation of its mixed form, Stokes' and Brinkman's equations. The constant in the corresponding abstract energy estimate is shown to be robust with respect to mesh parameters as well as the contrast, which is defined as the ratio of high and low values of the conductivity (or permeability). The derived stable decomposition allows to construct additive overlapping Schwarz iterative methods with condition numbers uniformly bounded with respect to the contrast and mesh parameters. The coarse spaces are obtained by patching together the eigenfunctions corresponding to the smallest eigenvalues of certain local problems. A detailed analysis of the abstract setting is provided. The proposed decomposition builds on a method of Galvis and Efendiev [Multiscale Model. Simul. 8 (2010) 1461-1483] developed for second order scalar elliptic problems with high contrast. Applications to the finite element discretizations of the second order elliptic problem in Galerkin and mixed formulation, the Stokes equations, and Brinkman's problem are presented. A number of numerical experiments for these problems in two spatial dimensions are provided.
\end{abstract}

Mathematics Subject Classification. 65F10, 65N20, 65N22, 65N30, 65 N55.

Received March 3, 2011. Revised October 11, 2011.

Published online February 22, 2012.

\section{INTRODUCTION}

Symmetric positive definite operators appear in the modeling of a variety of problems from environmental and engineering sciences, e.g. heat conduction in industrial (compound) media or fluid flow in natural subsurfaces. Two main challenges arising in the numerical solution of these problems are (1) the problem size due to spatial scales and (2) high-contrast due to large variations in the physical problem parameters. The latter e.g. concerns disparities in the thermal conductivities of the constituents of compound media or in the permeability field

\footnotetext{
Keywords and phrases. Domain decomposition, robust additive Schwarz preconditioner, spectral coarse spaces, high contrast, Brinkman's problem, multiscale problems.

1 Dept. Mathematics, Texas A\&M University, College Station, Texas 77843, USA. efendiev@math.tamu.edu; jugal@math.tamu.edu; lazarov@math.tamu.edu

2 Radon Institute for Computational and Applied Mathematics (RICAM), Altenberger Strasse 69, 4040 Linz, Austria.

joerg.willems@ricam. oeaw.ac.at
} 
of porous media. These variations frequently range over several orders of magnitude leading to high condition numbers of the corresponding discrete systems. Besides variations in physical parameters, the discretization parameters (e.g. mesh size) also lead to large condition numbers of the respective discrete systems.

Since in general high condition numbers entail poor performances of iterative solvers such as conjugate gradients (CG), the design of preconditioners addressing the size of the problem and the magnitude in variations of the problem parameters has received lots of attention in the numerical analysis community. The commonly used approaches include domain decomposition methods ( $c f$. e.g. $[19,26,27])$ and multilevel/multigrid algorithms $(c f$. e.g. $[3,15,29])$. For certain classes of problems, including the scalar elliptic case, these methods are successful in making the condition number of the preconditioned system independent of the size of the problem. However, the design of preconditioners that are robust with respect to variations in physical parameters is more challenging.

Improvements in the standard domain decomposition methods for the scalar elliptic equation, $-\nabla$. $(\kappa(\boldsymbol{x}) \nabla \phi)=f$, with a highly variable conductivity $\kappa(\boldsymbol{x})$ were made in the case of special arrangements of the highly conductive regions with respect to the coarse cells. The construction of preconditioners for these problems has been extensively studied in the last three decades (see e.g. $[7,13,18,19,27]$ ). In the context of domain decomposition methods, one can consider overlapping and nonoverlapping methods. It was shown that nonoverlapping domain decomposition methods converge independently of the contrast (e.g. [17,20] and [27], Sects. 6.4.4 and 10.2.4) when conductivity variations within coarse regions are bounded. The condition number bound for the preconditioned linear system using a two level overlapping domain decomposition method scales with the contrast, defined as

$$
\max _{\boldsymbol{x} \in \Omega} \kappa(\boldsymbol{x}) / \min _{\boldsymbol{x} \in \Omega} \kappa(\boldsymbol{x})
$$

where $\Omega$ is the domain. The overall condition number estimate also involves the ratio $H / \delta$, where $H$ is the coarse-mesh size and $\delta$ is the size of the overlap region. The estimate with respect to the ratio $H / \delta$ can be improved with the help of the small overlap trick (e.g. $[13,27])$. In this paper we focus on improving the contrast-dependent portion (given by (1.1)) of the condition number. A generous overlap will be used in our methods.

Classical arguments to estimate the preconditioned condition number of a two level overlapping domain decomposition method use weighted Poincaré inequalities of the form

$$
\int_{\omega} \kappa|\nabla \xi|^{2}\left(\psi-I_{0}^{\omega} \psi\right)^{2} \mathrm{~d} \boldsymbol{x} \leq C \int_{\omega} \kappa|\nabla \psi|^{2} \mathrm{~d} \boldsymbol{x}
$$

where $\omega$ is a local subdomain in the global domain $\Omega, \xi$ is a partition of unity function subordinate to $\omega$, and $\psi \in H^{1}(\omega)$. The operator $I_{0}^{\omega} \psi$ is a local representation of the function $\psi$ in the coarse space. The constant $C$ above appears in the final bound for the condition number of the operator. Many of the classical arguments that analyze overlapping domain decomposition methods for high contrast problems assume that $\frac{\max _{\boldsymbol{x} \in \omega} \kappa(\boldsymbol{x})}{\min _{\boldsymbol{x}} \in \omega \kappa(\boldsymbol{x})}$ is bounded. When only $|\nabla \xi|^{2}$ is bounded, inequality (1.2) can be obtained from a weighted Poincaré inequality whose constant is independent of the contrast. This weighted Poincaré inequality is not always valid, so a number of works were successful in addressing the question of when it holds. In [24], it was proven that the weighted Poincaré inequality holds for quasi-monotonic coefficients. The author obtains robust preconditioners for the case of quasi-monotonic coefficients. We note that recently, the concept of quasi-monotonic coefficient has been generalized in $[21,22]$ where the authors analyze nonoverlapping FETI methods. Other approaches use special partitions of unity such that the "pointwise energy" $\kappa(\boldsymbol{x})|\nabla \xi|^{2}$ in (1.2) is bounded. In [13, 28], the analysis in [27] has been extended to obtain explicit bounds involving the quantity $\kappa(\boldsymbol{x})|\nabla \xi|^{2}$. It has been shown that if the coarse space basis functions are constructed properly, then $\kappa(\boldsymbol{x})|\nabla \xi|^{2}$ remains bounded for all basis and partition of unity functions used, and then, the classical Poincaré inequality can be applied in the analysis. In particular, two main sets of coarse basis functions have been used: (1) multiscale finite element functions with various boundary conditions (see $[8,13,16]$ ) and $(2)$ energy minimizing or trace minimizing functions (see $[28,32]$ and references therein). Thus, robust overlapping domain decomposition methods can be constructed for 
the case when the high-conductivity regions are isolated islands. These methods use one coarse basis function per coarse node.

Recently, new coarse basis functions have been proposed in $[10,11]$. The construction of these coarse basis functions uses local generalized eigenvalue problems. The resulting methods can handle a general class of heterogeneous coefficients with high contrast. A main step in this construction is to identify those initial multiscale basis functions that are used to compute a weight function for the eigenvalue problem. These initial multiscale basis functions are designed to capture the effects that can be localized within coarse blocks. They are further complemented using generalized eigenfunctions that account for features of the solution that cannot be localized. The idea of using local and global eigenvectors to construct coarse spaces within two-level and multi-level techniques has been used before $(e . g .[6,25])$. However, these authors did not study the convergence with respect to physical parameters, such as high contrast and physical parameter variation, and did not use generalized eigenvalue problems to achieve small dimensional coarse spaces.

In many applications, the discretization technique is chosen so that it preserves the essential physical properties. For example, mixed finite element methods are often used in flow equations to obtain locally mass conservative velocity fields. In a number of flow applications, high-conductivity regions need to be represented with flow equations, such as Navier-Stokes' or Stokes' equations due to high porosity. Such complex systems can be described by Brinkman's equations that may require a special stable discretization. The method proposed in $[10,11]$ cannot be easily applied to vector problems and more complicated discretization methods. More precisely, it requires a proper eigenvalue problem for each particular differential equation.

In this paper, we extend the framework proposed in $[10,11]$ to general symmetric bilinear forms. The resulting analysis can be applied to a wide variety of differential equations that are important in practice. A key in designing robust preconditioners is a stable decomposition of the global function space into local and coarse subspaces (see [27]). This is the main focus of our paper. We develop an abstract framework that allows deriving a generalized eigenvalue problem for the construction of the coarse spaces and stable decompositions. In particular, some explicit bounds are obtained for the stability constant of this decomposition. In the scalar elliptic case, the analysis presented here leads to generalized eigenvalue problems that differ from those studied in $[10,11]$.

We consider the application of this abstract framework to Darcy's equation as well as to Brinkman's equations. Brinkman's equations can be viewed as a generalization of Darcy's equation that allows both Darcy and Stokes regions in the flow. Because Darcy's equation is obtained under the assumption of slow flow, Brinkman's equation is inherently high-contrast. It combines high flow described by Stokes' equations and slow flow described by Darcy's equation. In fact, the use of high conductivities in Darcy's equation may be associated to free flow or high porosity regions that are often described by Brinkman's equations (see [5]). The proposed general framework can be applied to the construction of robust coarse spaces for Brinkman's equations. These coarse spaces are constructed by passing to the stream function formulation. We note that in many applications forward problems are solved multiple times for different source terms and boundary conditions in the same media. In these problems, coarse spaces can be reused, which will decrease the computational cost.

In the paper at hand, we also discuss some coarse space dimension reduction techniques within our abstract framework. The dimension reduction is achieved by choosing initial multiscale basis functions that capture as much subgrid information as possible. We discuss how the choice of various initial multiscale basis functions affects the condition number of the preconditioned system.

To test the developed theory we consider several numerical examples. In our first set of numerical examples, we study elliptic equations with highly variable coefficients. We use both piecewise bilinear and multiscale basis functions as an initial coarse space. In the latter choice, they capture the effects of isolated high-conductivity regions. Our numerical results show that in both cases one obtains robust preconditioners. The use of multiscale basis functions as an initial coarse space allows substantial dimension reduction for problems with many small isolated inclusions. Both Galerkin and mixed formulations are studied in this paper. The next set of numerical results are on Brinkman's equations. These equations are discretized using $H$ (div)-conforming Discontinuous Galerkin methods. The numerical results show that the number of iterations is independent of the contrast. In our final numerical example, we consider a complex geometry with highly-variable coefficients without apparent 


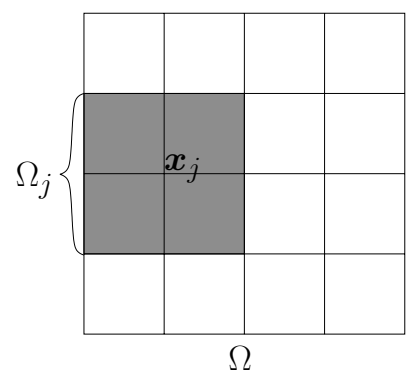

Figure 1. Triangulation $\mathcal{T}_{H}$.

separation of high and low conductivity regions. The numerical results show that using multiscale initial basis functions, one can obtain robust preconditioners with small coarse dimensional spaces.

The paper is organized as follows. In Section 2, problem setting and notation are introduced. In Section 3, the abstract analysis of the stable decomposition is presented. Section 4 is dedicated to applications of the abstract framework to the Galerkin and mixed formulation of Darcy's equation, Stokes' equations, and Brinkman's equations. In Section 5, we discuss the dimension reduction of the coarse space. Representative numerical results are presented in Section 6.

\section{Problem Setting And notation}

Let $\Omega \subset \mathbb{R}^{n}$ be a bounded polyhedral domain, and let $\mathcal{T}_{H}$ be a quasiuniform quadrilateral $(n=2)$ or hexahedral $(n=3)$ triangulation of $\Omega$ with mesh-parameter $H$. Let $\mathcal{X}=\left\{\boldsymbol{x}_{j}\right\}_{j=1}^{n_{\boldsymbol{x}}}$ be the set of nodes of $\mathcal{T}_{H}$, and for each $\boldsymbol{x}_{j} \in \mathcal{X}$ we set

$$
\Omega_{j}:=\text { interior }\left(\bigcup\left\{\bar{T} \mid T \in \mathcal{T}_{H}, \boldsymbol{x}_{j} \in \bar{T}\right\}\right),
$$

i.e., $\Omega_{j}$ is the union of all cells surrounding $\boldsymbol{x}_{j}$ (see Fig. 1). Define $I_{j}:=\left\{i=1, \ldots, n_{\boldsymbol{x}} \mid \Omega_{i} \cap \Omega_{j} \neq \emptyset\right\}$. Let $n_{I}:=\max _{j=1, \ldots, n_{x}} \# I_{j}$ denote the maximal number of overlaps of the $\Omega_{j}$ 's.

For a suitable finite dimensional space $\mathscr{V}_{0}=\mathscr{V}_{0}(\Omega)$ of functions defined on $\Omega$ and for any subdomain $\omega \subset \Omega$ we set $\mathscr{V}(\omega):=\left\{\left.\phi\right|_{\omega} \mid \phi \in \mathscr{V}_{0}\right\}$, and we consider a family of symmetric positive semi-definite bounded bilinear forms

$$
a_{\omega}(\cdot, \cdot):(\mathscr{V}(\omega), \mathscr{V}(\omega)) \rightarrow \mathbb{R} .
$$

We think of $\mathscr{V}_{0}$ as some standard finite element space. For the case $\omega=\Omega$ we drop the subindex, i.e., $a(\cdot, \cdot)=$ $a_{\Omega}(\cdot, \cdot)$ and we additionally assume that $a(\cdot, \cdot)$ is positive definite. For ease of notation we write $a_{\omega}(\phi, \psi)$ instead of $a_{\omega}\left(\left.\phi\right|_{\omega},\left.\psi\right|_{\omega}\right)$ for any $\phi, \psi \in \mathscr{V}_{0}$. Furthermore, we assume that for any $\phi, \psi \in \mathscr{V}_{0}$ we have

$$
\begin{gathered}
a(\phi, \phi) \leq \sum_{j=1}^{n_{x}} a_{\Omega_{j}}(\phi, \phi) \leq n_{I} a(\phi, \phi), \\
\left.a(\phi, \psi)=a_{\omega}(\phi, \psi), \text { if } \text { interior } \operatorname{supp}(\phi) \cap \operatorname{supp}(\psi)\right) \subset \omega, \\
a_{\omega}(\phi, \psi)=0, \text { if measure }(\omega)=0, \\
a_{\omega}(\phi, \phi) \leq a(\phi, \phi), \text { with equality if } \operatorname{supp}(\phi) \subset \omega .
\end{gathered}
$$


For an element $F$ in the dual space of $\mathscr{V}_{0}$, i.e., $F \in \mathscr{V}_{0}^{\prime}$, we consider the following variational formulation: find $\phi \in \mathscr{V}_{0}$ such that

$$
a(\phi, \psi)=F(\psi), \quad \text { for all } \psi \in \mathscr{V}_{0} .
$$

In operator notation this may be rewritten as

$$
A \phi=F .
$$

Let $\mathscr{V}_{0}\left(\Omega_{j}\right) \subset\left\{\psi \in \mathscr{V}_{0} \mid \operatorname{supp}(\psi) \subset \bar{\Omega}_{j}\right\}$ be a subspace of locally supported functions. Here and below we identify functions in $\left\{\psi \in \mathscr{V}_{0} \mid \operatorname{supp}(\psi) \subset \bar{\Omega}_{j}\right\}$ with their restrictions to $\Omega_{j}$. Thus, we in particular have $\mathscr{V}_{0}\left(\Omega_{j}\right) \subset$ $\mathscr{V}\left(\Omega_{j}\right)$. Note that $a_{\Omega_{j}}(\cdot, \cdot):\left(\mathscr{V}_{0}\left(\Omega_{j}\right), \mathscr{V}_{0}\left(\Omega_{j}\right)\right) \rightarrow \mathbb{R}$ is positive definite, whereas $a_{\Omega_{j}}(\cdot, \cdot):\left(\mathscr{V}\left(\Omega_{j}\right), \mathscr{V}\left(\Omega_{j}\right)\right) \rightarrow \mathbb{R}$ is in general only positive semi-definite.

Our main goal is the design of a "coarse" space $\mathscr{V}_{H}=\mathscr{V}_{H}(\Omega) \subset \mathscr{V}$ such that the overlapping additive Schwarz preconditioner $M: \mathscr{V}_{0}^{\prime} \rightarrow \mathscr{V}_{0}$ corresponding to $\left\{\mathscr{V}_{0}\left(\Omega_{j}\right)\right\}_{j=1}^{n_{x}}$ and $\mathscr{V}_{H}$ yields a robust condition number when applied to (2.2). The action of the overlapping additive Schwarz preconditioner corresponding to $\left\{\mathscr{V}_{0}\left(\Omega_{j}\right)\right\}_{j=1}^{n_{x}}$ and $\mathscr{V}_{H}$ is given by Algorithm 1 . For more details see e.g. [19,27].

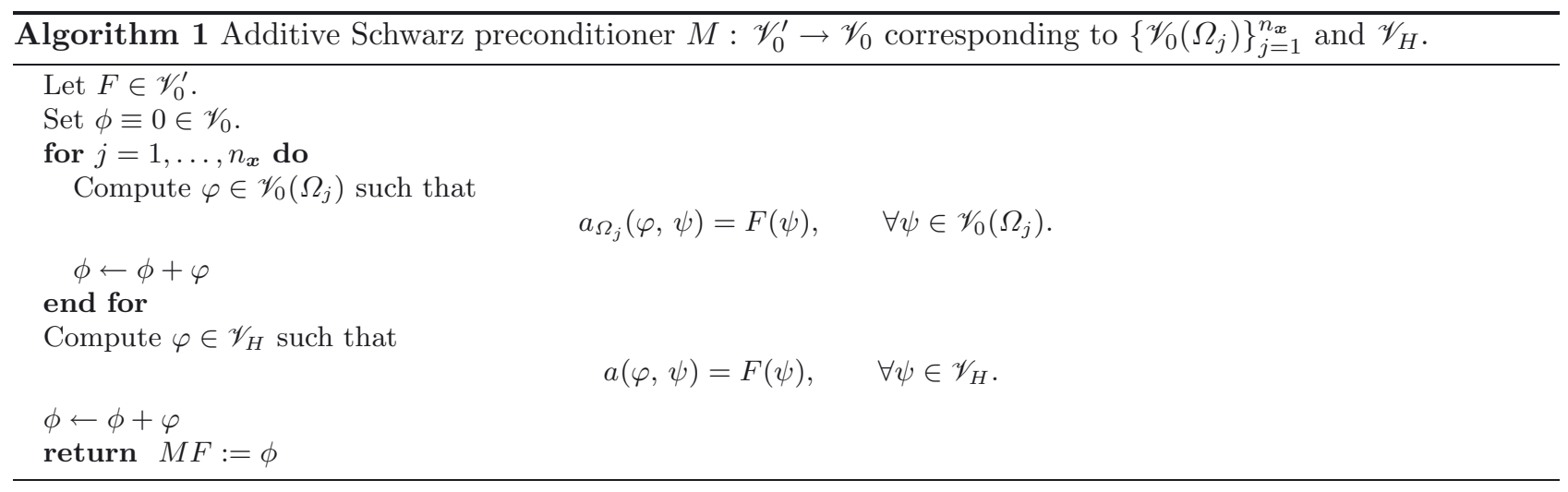

In order to obtain condition number bounds for the preconditioned system

$$
M A \phi=M F,
$$

the choice of the coarse space $\mathscr{V}_{H}$ is of fundamental importance. In particular, we will construct $\mathscr{V}_{H}$ with the following property: for any $\phi \in \mathscr{V}_{0}$ there is a representation

$$
\phi=\phi_{0}+\sum_{j=1}^{n_{x}} \phi_{j} \text { with } \phi_{0} \in \mathscr{V}_{H}, \text { and } \phi_{j} \in \mathscr{V}_{0}\left(\Omega_{j}\right) \text { for } j=1, \ldots, n_{\boldsymbol{x}}
$$

such that

$$
\sum_{j=0}^{n_{x}} a\left(\phi_{j}, \phi_{j}\right) \leq C a(\phi, \phi) .
$$

Let $\mathcal{E}=\left(\varepsilon_{i, j}\right)_{i, j=1}^{n_{\boldsymbol{x}}}$ with $\varepsilon_{i, j}=\left\{\begin{array}{c}1, \text { if } j \in I_{i} \\ 0 \text {, otherwise }\end{array}\right.$. By $(2.1 \mathrm{~b})$ and $(2.1 \mathrm{c})$ we then have for any $i, j=1, \ldots, n_{\boldsymbol{x}}$, $\phi \in \mathscr{V}_{0}\left(\Omega_{i}\right)$, and $\psi \in \mathscr{V}_{0}\left(\Omega_{j}\right)$ that $a(\phi, \psi) \leq \varepsilon_{i, j} a(\phi, \phi)^{1 / 2} a(\psi, \psi)^{1 / 2}$, i.e., $\mathcal{E}$ is the matrix of strengthened Cauchy-Schwarz inequality parameters. Note that $\max _{1 \leq i \leq n_{x}} \sum_{j=1}^{n_{x}} \varepsilon_{i, j}=n_{I}$. Thus, due to [19], Lemma 2.51, we have 
Theorem 2.1 (see Thm. 2.52 in [19]). The overlapping additive Schwarz preconditioner corresponding to the decomposition in (2.4a) yields a condition number of the preconditioned system (2.3) which is bounded by $C\left(n_{I}+1\right)$, with $C$ as in $(2.4 \mathrm{~b})$.

In view of Theorem 2.1 it is thus desirable to "control" the constant in (2.4b) and to keep the dimension of $\mathscr{V}_{H}$ "as small as possible".

For the construction of $\mathscr{V}_{H}$ we need more notation. Let $\left\{\xi_{j}\right\}_{j=1}^{n_{x}}: \Omega \rightarrow[0,1]$ be a partition of unity subordinate to $\left\{\Omega_{j}\right\}_{j=1}^{n_{x}}$ such that $\operatorname{supp}\left(\xi_{j}\right)=\bar{\Omega}_{j}$ and for any $\phi \in \mathscr{V}_{0}$ we have $\xi_{j} \phi \in \mathscr{V}_{0}\left(\Omega_{j}\right), j=1, \ldots, n_{\boldsymbol{x}}$. Using this notation we may for any $i, j=1, \ldots, n_{\boldsymbol{x}}$ define the following symmetric bilinear form:

$$
m_{\Omega_{j}}(\cdot, \cdot):\left(\mathscr{V}\left(\Omega_{j}\right), \mathscr{V}\left(\Omega_{j}\right)\right) \rightarrow \mathbb{R}, \quad m_{\Omega_{j}}(\phi, \psi):=\sum_{i \in I_{j}} a_{\Omega_{j}}\left(\xi_{j} \xi_{i} \phi, \xi_{j} \xi_{i} \psi\right)
$$

To ease notation, as we did for the bilinear form $a(\cdot, \cdot)$, we write $m_{\Omega_{j}}(\phi, \psi)$ instead of $m_{\Omega_{j}}\left(\left.\phi\right|_{\Omega_{j}},\left.\psi\right|_{\Omega_{j}}\right)$ for any $\phi, \psi \in \mathscr{V}_{0}$.

Due to our assumptions on $\left\{\xi_{j}\right\}_{j=1}^{n_{x}}$ we have that (2.5) is well-defined. Also note, that since $\operatorname{supp}\left(\xi_{j}\right)=\bar{\Omega}_{j}$ we have $\left.\xi_{j} \phi \equiv 0 \Leftrightarrow \phi\right|_{\Omega_{j}} \equiv 0$, which implies that $m_{\Omega_{j}}(\cdot, \cdot):\left(\mathscr{V}\left(\Omega_{j}\right), \mathscr{V}\left(\Omega_{j}\right)\right) \rightarrow \mathbb{R}$ is positive definite.

We point out that in Equation (2.5) we multiply by the partition of unity two times. In general, one of the multiplications cuts the support of the function to a coarse grid neighborhood and the next multiplication cuts the support of the function to a subdomain where a local solver is implemented. A more general version of (2.5) can be considered where two different partition of unity functions are used (as in [10,11]). Using two different partitions of unity allows us to treat the case where the coarse grid and the local solvers subdomains are not related. Our analysis easily carries over to the case of using different partitions of unity.

Now for $j=1, \ldots, n_{\boldsymbol{x}}$ we consider the generalized eigenvalue problems: Find $\left(\lambda_{i}^{j}, \varphi_{i}^{j}\right) \in\left(\mathbb{R}, \mathscr{V}\left(\Omega_{j}\right)\right)$ such that

$$
a_{\Omega_{j}}\left(\psi, \varphi_{i}^{j}\right)=\lambda_{i}^{j} m_{\Omega_{j}}\left(\psi, \varphi_{i}^{j}\right), \quad \forall \psi \in \mathscr{V}\left(\Omega_{j}\right)
$$

Without loss of generality we assume that the eigenvalues are ordered, i.e., $0 \leq \lambda_{1}^{j} \leq \lambda_{2}^{j} \leq \ldots \leq \lambda_{i}^{j} \leq \lambda_{i+1}^{j} \leq \ldots$

It is easy to see that any two eigenfunctions corresponding to two distinct eigenvalues are $a_{\Omega_{j}}(\cdot, \cdot)$ - and $m_{\Omega_{j}}(\cdot, \cdot)$-orthogonal. By orthogonalizing the eigenfunctions corresponding to the same eigenvalues we can thus, without loss of generality, assume that all computed eigenfunctions are pairwisely $a_{\Omega_{j}}(\cdot, \cdot)$ - and $m_{\Omega_{j}}(\cdot, \cdot)$ orthogonal. Now, every function in $\mathscr{V}\left(\Omega_{j}\right)$ has an expansion with respect to the eigenfunctions of (2.6). This is the reason why the generalized eigenproblem is posed with respect to $\mathscr{V}\left(\Omega_{j}\right)$ as opposed to $\mathscr{V}_{0}\left(\Omega_{j}\right)$.

For an arbitrary "threshold" $\tau_{\lambda}^{-1}>0$ we choose $L_{j}$ such that $\lambda_{L_{j}+1}^{j} \geq \tau_{\lambda}^{-1}$. We prefer to use the inverse of $\tau_{\lambda}$ here, since in the estimates below the inverse of the threshold appears. For $\phi \in \mathscr{V}_{0}$ let $\phi_{0}^{j}$ be the $m_{\Omega_{j}}(\cdot, \cdot)$ orthogonal projection of $\left.\phi\right|_{\Omega_{j}}$ onto the first $L_{j}$ eigenfunctions of (2.6), i.e.,

$$
m_{\Omega_{j}}\left(\phi-\phi_{0}^{j}, \varphi_{i}^{j}\right)=0, \quad \forall i=1, \ldots, L_{j}
$$

If $L_{j}=0$, we set $\phi_{0}^{j} \equiv 0$. Since any function in $\mathscr{V}\left(\Omega_{j}\right)$ has an expansion with respect to the eigenfunctions of $(2.6)$ we can now choose $\alpha_{i} \in \mathbb{R}$ so that

$$
\left.\phi\right|_{\Omega_{j}}-\phi_{0}^{j}=\sum_{i>L_{j}} \alpha_{i} \varphi_{i}^{j} .
$$


Then we observe that

$$
\begin{aligned}
m_{\Omega_{j}}\left(\phi-\phi_{0}^{j}, \phi-\phi_{0}^{j}\right) & =m_{\Omega_{j}}\left(\sum_{i>L_{j}} \alpha_{i} \varphi_{i}^{j}, \sum_{i>L_{j}} \alpha_{i} \varphi_{i}^{j}\right) \\
& =\sum_{i>L_{j}} m_{\Omega_{j}}\left(\alpha_{i} \varphi_{i}^{j}, \alpha_{i} \varphi_{i}^{j}\right) \quad \text { (by orthogonality) } \quad \text { (by (2.6)) } \\
& =\sum_{i>L_{j}} \frac{1}{\lambda_{i}^{j}} a_{\Omega_{j}}\left(\alpha_{i} \varphi_{i}^{j}, \alpha_{i} \varphi_{i}^{j}\right) \quad \\
& \leq \frac{1}{\lambda_{L_{j}+1}^{j}} \sum_{i>L_{j}} a_{\Omega_{j}}\left(\alpha_{i} \varphi_{i}^{j}, \alpha_{i} \varphi_{i}^{j}\right) \\
& \leq \tau_{\lambda} a_{\Omega_{j}}\left(\phi-\phi_{0}^{j}, \phi-\phi_{0}^{j}\right) \\
& \leq \tau_{\lambda} a_{\Omega_{j}}(\phi, \phi) .
\end{aligned}
$$

\section{Coarse spaces yielding Robust stable DeCompositions}

With these preliminaries we are now able to define a decomposition described in (2.4): first, we specify the coarse space by

$$
\mathscr{V}_{H}:=\operatorname{span}\left\{\xi_{j} \varphi_{i}^{j} \mid j=1, \ldots, n_{\boldsymbol{x}} \text { and } i=1, \ldots, L_{j}\right\}
$$

Then, for any $\phi \in \mathscr{V}$ let

$$
\phi_{0}:=\sum_{j=1}^{n_{x}} \xi_{j} \phi_{0}^{j} \in \mathscr{V}_{H}
$$

where $\phi_{0}^{j}$ is chosen according to $(2.7)$. For $j=1, \ldots, n_{\boldsymbol{x}}$ define

$$
\phi_{j}:=\xi_{j}\left(\phi-\phi_{0}\right) \in \mathscr{V}_{0}\left(\Omega_{j}\right) \quad \text { so that } \quad \phi=\sum_{j=0}^{n_{x}} \phi_{j} .
$$

Before analyzing this decomposition we summarize all assumptions using the notation above:

$(\mathrm{A} 1) a_{\omega}(\cdot, \cdot):(\mathscr{V}(\omega), \mathscr{V}(\omega)) \rightarrow \mathbb{R}$ is symmetric positive semi-definite for any subdomain $\omega \subset \Omega$, and $a(\cdot, \cdot)=$ $a_{\Omega}(\cdot, \cdot)$ is positive definite.

(A2) For any $\phi, \psi \in \mathscr{V}_{0}$ we have that (2.1) holds.

(A3) The local subspaces $\mathscr{V}_{0}\left(\Omega_{j}\right), j=1, \ldots, n_{\boldsymbol{x}}$ satisfy $\mathscr{V}_{0}\left(\Omega_{j}\right) \subset\left\{\psi \in \mathscr{V}_{0} \mid \operatorname{supp}(\psi) \subset \bar{\Omega}_{j}\right\}$.

(A4) $\left\{\xi_{j}\right\}_{j=1}^{n_{x}}: \Omega \rightarrow[0,1]$ is a family of functions such that

(a) $\sum_{j=1}^{n_{x}} \xi_{j} \equiv 1$ on $\Omega$;

(b) $\operatorname{supp}\left(\xi_{j}\right)=\bar{\Omega}_{j}$ for $j=1, \ldots, n_{\boldsymbol{x}}$;

(c) for any $\phi \in \mathscr{V}_{0}$ we have $\xi_{j} \phi \in \mathscr{V}_{0}\left(\Omega_{j}\right)$ for any $j=1, \ldots, n_{\boldsymbol{x}}$.

As noted above, these assumptions imply in particular, that for any $1 \leq j \leq n_{\boldsymbol{x}}$, the bilinear form $m_{\Omega_{j}}(\cdot, \cdot)$ : $\left(\mathscr{V}\left(\Omega_{j}\right), \mathscr{V}\left(\Omega_{j}\right)\right) \rightarrow \mathbb{R}$ is positive definite.

Remark 3.1. Considering infinite dimensional function spaces assumption (A4)(c) is reasonable in many situations (see Sects. 4 and 5). However, when $\mathscr{V}_{0}\left(\Omega_{j}\right)$ is a standard finite element space assumption (A4)(c) is unrealistic. Generally, the product of a partition of unity function $\xi_{j}$ and a finite element function $\phi$ does not belong to the same finite element space as $\phi$. There are two ways to overcome this problem: (1) to project $\xi_{j} \phi$ back to the finite element space using the form $a(\cdot, \cdot)$ or $(2)$ to use the finite element interpolant of $\xi_{j} \phi$. 
(1) has the advantage that the energy of the $a(\cdot, \cdot)$-orthogonal projection of $\xi_{j} \phi$ is bounded by the energy of $\xi_{j} \phi$ itself. This result holds in the generality of the setting which we have hitherto assumed. Nevertheless, even though the projection is a local operation, it involves inverting some local stiffness matrices and could thus be unnecessarily computationally expensive;

(2) has the advantage that it can be implemented straightforwardly at negligible computational cost. The downside of this approach, however, is that it is difficult to obtain energy estimates as for (1) in the generality of our present setting. At best, one can expect to establish that the energy of the interpolant of $\xi_{j} \phi$ is bounded by the energy of $\xi_{j} \phi$ in a very concrete setting. Thus, (2) entails a loss of generality that needs to be compensated by analyzing the particular setting, i.e., the choice of the finite element space and of the partition of unity functions; see [11] for the scalar elliptic case with piecewise linear elements.

We use approach (2) in our numerical computations (see Sect. 6).

The following theorem is the main theoretical result of our paper. It establishes a stable decomposition with a constant only depending on $n_{I}$ and $\tau_{\lambda}$.

Theorem 3.2. Assume (A1)-(A4) hold. Then, the decomposition defined in (3.2) satisfies

$$
\sum_{j=0}^{n_{x}} a\left(\phi_{j}, \phi_{j}\right) \leq\left(2+C \tau_{\lambda}\right) a(\phi, \phi),
$$

where $C$ only depends on $n_{I}$.

Combining Theorems 3.2 and 2.1 we immediately obtain the following

Corollary 3.3. Assuming that (A1)-(A4) hold, the additive Schwarz preconditioner corresponding to the decomposition in (3.2) yields a condition number of the preconditioned system (2.3) which is bounded by $C\left(1+\tau_{\lambda}\right)$, where $C$ only depends on $n_{I}$.

The proof of Theorem 3.2 is split into the following two lemmas establishing the required energy bounds for the local contributions and the coarse contribution in decomposition (2.4), respectively.

Lemma 3.4. Assume (A1)-(A4) hold. Then, for $\phi \in \mathscr{V}_{0}$ we have

$$
\sum_{j=1}^{n_{x}} a\left(\phi_{j}, \phi_{j}\right) \leq C \tau_{\lambda} a(\phi, \phi),
$$

where $C$ only depends on $n_{I}$ (the maximal number of overlaps of the subdomains $\left\{\Omega_{j}\right\}_{j=1}^{n_{x}}$ ).

Proof. Observe that

$$
\begin{aligned}
\sum_{j=1}^{n_{x}} a\left(\phi_{j}, \phi_{j}\right) & =\sum_{j=1}^{n_{x}} a_{\Omega_{j}}\left(\xi_{j}\left(\phi-\phi_{0}\right), \xi_{j}\left(\phi-\phi_{0}\right)\right) \\
& =\sum_{j=1}^{n_{x}} a_{\Omega_{j}}\left(\xi_{j} \sum_{i=1}^{n_{x}} \xi_{i}\left(\phi-\phi_{0}^{i}\right), \xi_{j} \sum_{i=1}^{n_{x}} \xi_{i}\left(\phi-\phi_{0}^{i}\right)\right) \\
& =\sum_{j=1}^{n_{x}} a_{\Omega_{j}}\left(\xi_{j} \sum_{i \in I_{j}} \xi_{i}\left(\phi-\phi_{0}^{i}\right), \xi_{j} \sum_{i \in I_{j}} \xi_{i}\left(\phi-\phi_{0}^{i}\right)\right) \\
& \leq n_{I} \underbrace{n_{j=1}^{n_{x}} \sum_{i \in I_{j}} a_{\Omega_{j}}\left(\xi_{j} \xi_{i}\left(\phi-\phi_{0}^{i}\right), \xi_{j} \xi_{i}\left(\phi-\phi_{0}^{i}\right)\right)}_{=: E_{1}},
\end{aligned}
$$


where in the last step we have used Schwarz' inequality together with $\# I_{j} \leq n_{I}$. Note, that we furthermore have

$$
\begin{array}{rlr}
E_{1} & =\sum_{j=1}^{n_{x}} \sum_{i \in I_{j}} a_{\Omega_{i}}\left(\xi_{j} \xi_{i}\left(\phi-\phi_{0}^{i}\right), \xi_{j} \xi_{i}\left(\phi-\phi_{0}^{i}\right)\right)(\text { by }(\mathrm{A} 2)) \\
& =\sum_{i=1}^{n_{x}} \sum_{j \in I_{i}} a_{\Omega_{i}}\left(\xi_{j} \xi_{i}\left(\phi-\phi_{0}^{i}\right), \xi_{j} \xi_{i}\left(\phi-\phi_{0}^{i}\right)\right) \\
& =\sum_{i=1}^{n_{x}} m_{\Omega_{i}}\left(\phi-\phi_{0}^{i}, \phi-\phi_{0}^{i}\right) . & \quad(\text { by }(2.5))
\end{array}
$$

Thus, we obtain

$$
\begin{aligned}
\sum_{j=1}^{n_{x}} a\left(\phi_{j}, \phi_{j}\right) & \leq n_{I} \sum_{j=1}^{n_{x}} m_{\Omega_{j}}\left(\phi-\phi_{0}^{j}, \phi-\phi_{0}^{j}\right) \\
& \leq n_{I} \tau_{\lambda} \sum_{j=1}^{n_{x}} a_{\Omega_{j}}(\phi, \phi) \\
& \leq n_{I}^{2} \tau_{\lambda}(\phi, \phi)
\end{aligned}
$$

where the last inequality holds due to (A2).

Remark 3.5. Note, that by the proof of Lemma 3.4 we in particular have

$$
\sum_{j=1}^{n_{x}} a_{\Omega_{j}}\left(\xi_{j} \sum_{i \in I_{j}} \xi_{i}\left(\phi-\phi_{0}^{i}\right), \xi_{j} \sum_{i \in I_{j}} \xi_{i}\left(\phi-\phi_{0}^{i}\right)\right) \leq n_{I}^{2} \tau_{\lambda} a(\phi, \phi) .
$$

Now, we proceed with the necessary energy estimate for the coarse component $\phi_{0}$ of the decomposition (3.2a).

Lemma 3.6. Let (A1)-(A4) hold. Then, for $\phi_{0}$ defined by (3.2a) we have that

$$
a\left(\phi_{0}, \phi_{0}\right) \leq\left(2+C \tau_{\lambda}\right) a(\phi, \phi),
$$

where, as above, $C$ only depends on $n_{I}$.

Proof. First, we note that

$$
\begin{aligned}
a\left(\phi_{0}, \phi_{0}\right) & =a\left(\sum_{i=1}^{n_{x}} \xi_{i} \phi_{0}^{i}, \sum_{i=1}^{n_{x}} \xi_{i} \phi_{0}^{i}\right) \\
& \left.=a\left(\sum_{i=1}^{n_{x}} \xi_{i}\left(\phi_{0}^{i}-\phi\right)+\phi, \sum_{i=1}^{n_{x}} \xi_{i}\left(\phi_{0}^{i}-\phi\right)+\phi\right) \quad \text { by }(3.2 \mathrm{a})\right) \\
& \leq 2 \underbrace{\left(\sum_{i=1}^{n_{x}} \xi_{i}\left(\phi_{0}^{i}-\phi\right), \sum_{i=1}^{n_{x}} \xi_{i}\left(\phi_{0}^{i}-\phi\right)\right)}_{=: E_{2}}+2 a(\phi, \phi)
\end{aligned}
$$


where we have used Schwarz' inequality. Now, observe that

$$
\begin{aligned}
E_{2} \leq & \sum_{j=1}^{n_{x}} a_{\Omega_{j}}\left(\sum_{i \in I_{j}} \xi_{i}\left(\phi_{0}^{i}-\phi\right), \sum_{i \in I_{j}} \xi_{i}\left(\phi_{0}^{i}-\phi\right)\right) \\
= & \sum_{j=1}^{n_{\boldsymbol{x}}} a_{\Omega_{j}}\left(\left(1-\xi_{j}+\xi_{j}\right) \sum_{i \in I_{j}} \xi_{i}\left(\phi_{0}^{i}-\phi\right),\left(1-\xi_{j}+\xi_{j}\right) \sum_{i \in I_{j}} \xi_{i}\left(\phi_{0}^{i}-\phi\right)\right) \\
\leq & 2 \underbrace{\sum_{j=1}^{n_{x}} a_{\Omega_{j}}\left(\left(1-\xi_{j}\right) \sum_{i \in I_{j}} \xi_{i}\left(\phi_{0}^{i}-\phi\right),\left(1-\xi_{j}\right) \sum_{i \in I_{j}} \xi_{i}\left(\phi_{0}^{i}-\phi\right)\right)}_{=: E_{3}} \\
& +2 \sum_{j=1}^{n_{x}} a_{\Omega_{j}}\left(\xi_{j} \sum_{i \in I_{j}} \xi_{i}\left(\phi_{0}^{i}-\phi\right), \xi_{j} \sum_{i \in I_{j}} \xi_{i}\left(\phi_{0}^{i}-\phi\right)\right) \\
\leq & 2 E_{3}+2 n_{I}^{2} \tau_{\lambda} a(\phi, \phi),
\end{aligned}
$$

where we have again used Schwarz' inequality. Note, that

$$
\begin{aligned}
E_{3} & =\sum_{j=1}^{n_{x}} a_{\Omega_{j}}\left(\sum_{l \in I_{j} \backslash\{j\}} \xi_{l} \sum_{i \in I_{j}} \xi_{i}\left(\phi_{0}^{i}-\phi\right), \sum_{l \in I_{j} \backslash\{j\}} \xi_{l} \sum_{i \in I_{j}} \xi_{i}\left(\phi_{0}^{i}-\phi\right)\right) \quad \text { (by (A4)) } \\
& \leq n_{I} \sum_{j=1}^{n_{x}} \sum_{l \in I_{j} \backslash\{j\}} a_{\Omega_{j}}\left(\xi_{l} \sum_{i \in I_{j}} \xi_{i}\left(\phi_{0}^{i}-\phi\right), \xi_{l} \sum_{i \in I_{j}} \xi_{i}\left(\phi_{0}^{i}-\phi\right)\right) . \quad \text { (by Schwarz' inequality) }
\end{aligned}
$$

Since by (A2)

$$
\begin{aligned}
a_{\Omega_{j}}\left(\xi_{l} \sum_{i \in I_{j}} \xi_{i}\left(\phi_{0}^{i}-\phi\right), \xi_{l} \sum_{i \in I_{j}} \xi_{i}\left(\phi_{0}^{i}-\phi\right)\right) & =a_{\Omega_{j} \cap \Omega_{l}}\left(\xi_{l} \sum_{i \in I_{l}} \xi_{i}\left(\phi_{0}^{i}-\phi\right), \xi_{l} \sum_{i \in I_{l}} \xi_{i}\left(\phi_{0}^{i}-\phi\right)\right) \\
& \leq a_{\Omega_{l}}\left(\xi_{l} \sum_{i \in I_{l}} \xi_{i}\left(\phi_{0}^{i}-\phi\right), \xi_{l} \sum_{i \in I_{l}} \xi_{i}\left(\phi_{0}^{i}-\phi\right)\right),
\end{aligned}
$$

we thus have

$$
\begin{aligned}
E_{3} & \leq n_{I} \sum_{j=1}^{n_{x}} \sum_{l \in I_{j} \backslash\{j\}} a_{\Omega_{l}}\left(\xi_{l} \sum_{i \in I_{l}} \xi_{i}\left(\phi_{0}^{i}-\phi\right), \xi_{l} \sum_{i \in I_{l}} \xi_{i}\left(\phi_{0}^{i}-\phi\right)\right) & \\
& =n_{I} \sum_{l=1}^{n_{x}} \sum_{j \in I_{l} \backslash\{l\}} a_{\Omega_{l}}\left(\xi_{l} \sum_{i \in I_{l}} \xi_{i}\left(\phi_{0}^{i}-\phi\right), \xi_{l} \sum_{i \in I_{l}} \xi_{i}\left(\phi_{0}^{i}-\phi\right)\right) & \\
& \leq n_{I}^{2} \sum_{l=1}^{n_{x}} a_{\Omega_{l}}\left(\xi_{l} \sum_{i \in I_{l}} \xi_{i}\left(\phi_{0}^{i}-\phi\right), \xi_{l} \sum_{i \in I_{l}} \xi_{i}\left(\phi_{0}^{i}-\phi\right)\right) & \left(\#\left(I_{l} \backslash\{l\}\right) \leq n_{I}\right) \\
& \leq n_{I}^{4} \tau_{\lambda} a(\phi, \phi) . & \text { (by Rem. 3.5) }
\end{aligned}
$$

Combining (3.7), (3.8), and (3.9) we obtain

$$
a\left(\phi_{0}, \phi_{0}\right) \leq\left(2+4\left(n_{I}^{4}+n_{I}^{2}\right) \tau_{\lambda}\right) a(\phi, \phi) .
$$

Combining Lemmas 3.4 and 3.6 we obtain Theorem 3.2. 


\section{Applications}

To apply the theory developed in Sections 2 and 3 to some particular problem we need to verify assumptions (A1)-(A4). As indicated in Remark 3.1 we do this in the setting of infinite dimensional spaces. For standard finite element spaces assumption (A4)(c) is in general not satisfied, but this may be addressed as outlined in Remark 3.1. Having established assumptions (A1)-(A4), Corollary 3.3 yields that the condition number of the corresponding additive Schwarz preconditioned system has an upper bound which depends only on $n_{I}$ and $\tau_{\lambda}$.

In addition to verifying assumptions (A1)-(A4) we also have to make sure that the number of "small" eigenvalues in (2.6), i.e., those below the threshold $\tau_{\lambda}^{-1}$, and thus $\operatorname{dim}\left(\mathscr{V}_{H}\right)$, is significantly smaller than $\operatorname{dim}\left(\mathscr{V}_{0}\right)$, in order for our method to be practically beneficial. Note, that the choice of $\tau_{\lambda}^{-1}>0$ and thus the number of small eigenvalues is not unique. Nevertheless, for a certain choice of $\tau_{\lambda}^{-1}$ and a given problem we may still aim to establish that the number of eigenvalues below the chosen threshold is uniformly bounded with respect to changes in specific problem parameters. In this case the additive Schwarz preconditioner corresponding to the stable decomposition (3.2) has a coarse space whose dimension is uniformly bounded with respect to these parameters.

\subsection{The scalar elliptic case - Galerkin formulation}

As a first application of the abstract framework developed above, we consider the scalar elliptic equation

$$
-\nabla \cdot(\kappa \nabla \phi)=f, \quad \boldsymbol{x} \in \Omega, \text { and } \quad \phi=0, \quad \boldsymbol{x} \in \partial \Omega,
$$

where $\kappa \in L^{\infty}(\Omega)$ is a positive function, which may have large variations, $\phi \in H_{0}^{1}(\Omega)$, and $f \in L^{2}(\Omega)$. Note that we can always reduce to the case of homogeneous boundary conditions by introducing an appropriate right hand side.

With $\mathscr{V}_{0}:=H_{0}^{1}(\Omega)$, the variational formulation corresponding to (4.1) is: find $\phi \in \mathscr{V}_{0}$ such that for all $\psi \in \mathscr{V}_{0}$

$$
a^{S E}(\phi, \psi):=\int_{\Omega} \kappa(\boldsymbol{x}) \nabla \phi \cdot \nabla \psi \mathrm{d} \boldsymbol{x}=\int_{\Omega} f \psi \mathrm{d} \boldsymbol{x} .
$$

Let $\mathscr{V}_{0}\left(\Omega_{j}\right):=H_{0}^{1}\left(\Omega_{j}\right)$ for any $j=1, \ldots, n_{\boldsymbol{x}}$. Choosing $\left\{\xi_{j}\right\}_{j=1}^{n_{x}}$ the Lagrange finite element functions of degree one corresponding to $\mathcal{T}_{H}$, we readily see that for the scalar elliptic case, i.e., when setting $a(\cdot, \cdot)=a^{S E}(\cdot, \cdot)$, assumptions (A1)-(A4) are satisfied.

Let us for now assume that $\kappa$ assumes only two values. More precisely,

$$
\kappa(\boldsymbol{x})=\left\{\begin{array}{l}
\kappa_{\max } \text { in } \Omega^{s} \\
\kappa_{\min } \text { in } \Omega^{p},
\end{array} \text { with } \bar{\Omega}^{s} \cup \bar{\Omega}^{p}=\bar{\Omega} \text { and } \kappa_{\max } \gg \kappa_{\min }>0 .\right.
$$

Without loss of generality, we may take $\kappa_{\min }=1$. Now we wish to establish the existence of $\tau_{\lambda}^{-1}$ such that the number of eigenvalues below this threshold is independent of the contrast $\kappa_{\max } / \kappa_{\min }$, which is the problem parameter of interest in this situation.

By the well-known min-max principle [23], we know that the $i$-th eigenvalue of (2.6) is given by

$$
\lambda_{i}^{j}=\min _{\mathscr{V}_{i}\left(\Omega_{j}\right)} \max _{\psi \in \mathscr{V}_{i}\left(\Omega_{j}\right)} \frac{a_{\Omega_{j}}^{S E}(\psi, \psi)}{m_{\Omega_{j}}^{S E}(\psi, \psi)},
$$

where $\mathscr{V}_{i}\left(\Omega_{j}\right)$ is any $i$-dimensional subspace of $\mathscr{V}\left(\Omega_{j}\right)$.

We denote $\Omega_{j}^{s}:=\Omega^{s} \cap \Omega_{j}$ and $\Omega_{j}^{p}:=\Omega^{p} \cap \Omega_{j}$. Now, for each $i=1, \ldots, L_{j}$ let $\Omega_{j, i}^{s}$ denote the $i$-th connected component of $\Omega_{j}^{s}$, where $L_{j}$ denotes the total number of connected components of $\Omega_{j}^{s}$ (see Fig. 2). If $\Omega_{j}^{s}=\emptyset$, we set $\Omega_{j, 1}^{s}=\Omega_{j}$ and $L_{j}=1$. 


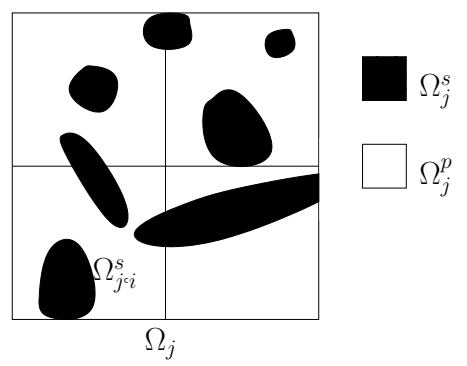

Figure 2. Subdomain with connected components of $\Omega_{j}^{s}$. In the present configuration $L_{j}=7$.

Now, we define the following subspace of $\mathscr{V}\left(\Omega_{j}\right)$ :

$$
\mathscr{V}_{S E}^{c}\left(\Omega_{j}\right):=\left\{\phi \in \mathscr{V}\left(\Omega_{j}\right) \mid \int_{\Omega_{j, i}^{s}} \phi \mathrm{d} \boldsymbol{x}=0, \text { for } i=1, \ldots, L_{j}\right\} .
$$

It is straightforward to see that any $L_{j}+1$-dimensional subspace of $\mathscr{V}\left(\Omega_{j}\right)$ has a non-trivial intersection with $\mathscr{V}_{S E}^{c}\left(\Omega_{j}\right)$. Thus, by (4.2) there exists a non-zero $\phi \in \mathscr{V}_{S E}^{c}\left(\Omega_{j}\right)$ such that

$$
\lambda_{L_{j}+1}^{j} \geq \frac{a_{\Omega_{j}}^{S E}(\phi, \phi)}{m_{\Omega_{j}}^{S E}(\phi, \phi)} .
$$

Using the definitions of $a_{\Omega_{j}}^{S E}(\cdot, \cdot)$ and $m_{\Omega_{j}}^{S E}(\cdot, \cdot)$ we note that

$$
\begin{aligned}
m_{\Omega_{j}}^{S E}(\phi, \phi) & =\sum_{i \in I_{j}} a_{\Omega_{j}}^{S E}\left(\xi_{j} \xi_{i} \phi, \xi_{j} \xi_{i} \phi\right) \\
& =\sum_{i \in I_{j}} \int_{\Omega_{j}} \kappa\left|\nabla\left(\xi_{j} \xi_{i} \phi\right)\right|^{2} \mathrm{~d} \boldsymbol{x} \\
& \leq 2 \sum_{i \in I_{j}} \int_{\Omega_{j}} \kappa\left(\left|\nabla\left(\xi_{j} \xi_{i}\right) \phi\right|^{2}+\left|\xi_{j} \xi_{i} \nabla \phi\right|^{2}\right) \mathrm{d} \boldsymbol{x} \\
& \leq C \sum_{i \in I_{j}} \int_{\Omega_{j}} \kappa\left(\left(H^{-1} \phi\right)^{2}+|\nabla \phi|^{2}\right) \mathrm{d} \boldsymbol{x} \\
& \leq C n_{I}\left(H^{-2} \int_{\Omega_{j}} \kappa \phi^{2} \mathrm{~d} \boldsymbol{x}+a_{\Omega_{j}}^{S E}(\phi, \phi)\right),
\end{aligned}
$$

where $C$ only depends on the choice of the partition of unity. Furthermore, we observe that $\int_{\Omega_{j}^{p}} \phi^{2} \mathrm{~d} \boldsymbol{x} \leq$ $\int_{\Omega_{j}} \phi^{2} \mathrm{~d} \boldsymbol{x} \leq C H^{2} \int_{\Omega_{j}}|\nabla \phi|^{2} \mathrm{~d} \boldsymbol{x} \leq C H^{2} \int_{\Omega_{j}} \kappa|\nabla \phi|^{2} \mathrm{~d} \boldsymbol{x}$, where the second inequality follows from Poincaré's inequality, which is possible since $\phi \in \mathscr{V}_{S E}^{c}\left(\Omega_{j}\right)$, and where the last inequality holds since $\kappa \geq 1$. Thus, we have

$$
\begin{aligned}
\int_{\Omega_{j}} \kappa \phi^{2} \mathrm{~d} \boldsymbol{x} & =\int_{\Omega_{j}^{p}} \phi^{2} \mathrm{~d} \boldsymbol{x}+\kappa_{\max } \sum_{i=1}^{L_{j}} \int_{\Omega_{j, i}^{s}} \phi^{2} \mathrm{~d} \boldsymbol{x} \\
& \leq C H^{2}\left(\int_{\Omega_{j}} \kappa|\nabla \phi|^{2} \mathrm{~d} \boldsymbol{x}+\kappa_{\max } \sum_{i=1}^{L_{j}} \int_{\Omega_{j, i}^{s}}|\nabla \phi|^{2} \mathrm{~d} \boldsymbol{x}\right) \\
& \leq C H^{2} \int_{\Omega_{j}} \kappa|\nabla \phi|^{2} \mathrm{~d} \boldsymbol{x},
\end{aligned}
$$


where for the first inequality we have also used Poincaré's inequality for the second summand, which again holds since $\phi \in \mathscr{V}_{S E}^{c}\left(\Omega_{j}\right)$. Here, $C$ is a constant which only depends on the geometries of $\Omega_{j}^{p}$ and $\Omega_{j, i}^{s}, i=1, \ldots, L_{j}$. Thus, we obtain

$$
m_{\Omega_{j}}^{S E}(\phi, \phi) \leq C a_{\Omega_{j}}^{S E}(\phi, \phi),
$$

which together with (4.4) yields a uniform (with respect to $\kappa_{\max } / \kappa_{\min }$ and $H$ ) lower bound for $\lambda_{L_{j}+1}^{j}$. Thus, we see that for a suitably chosen $\tau_{\lambda}$ the number of generalized eigenvalues below $\tau_{\lambda}^{-1}$ and satisfying (2.6) is bounded from above by the number of connected components in $\Omega_{j}^{s}$, i.e., $L_{j}$.

\subsection{The scalar elliptic case - mixed formulation}

In this Section we consider the mixed formulation of the scalar elliptic equation, also known as Darcy's equations, in 2 spatial dimensions, i.e., $n=2$, modeling flow in porous media

$$
\left\{\begin{array}{l}
\nabla p+\mu \kappa^{-1} \boldsymbol{u}=\boldsymbol{f} \quad \text { in } \Omega, \\
\nabla \cdot \boldsymbol{u}=0 \quad \text { in } \Omega, \\
\boldsymbol{u} \cdot \boldsymbol{n}=0 \quad \text { on } \partial \Omega .
\end{array}\right.
$$

Here, $p \in L_{0}^{2}(\Omega):=L^{2}(\Omega) / \mathbb{R}$ denotes the pressure, $\boldsymbol{u} \in H($ div $\Omega):=\left\{\boldsymbol{v} \in\left(L^{2}(\Omega)\right)^{2} \mid \nabla \cdot \boldsymbol{v} \in L^{2}(\Omega)\right\}$ is the velocity, $\boldsymbol{f} \in\left(L^{2}(\Omega)\right)^{2}$ is a forcing term, and $\boldsymbol{n}$ denotes the unit outer normal vector to $\partial \Omega$. The viscosity $\mu$ is a positive constant, and $\kappa \in L^{\infty}(\Omega)$ is a positive function. With $H_{0}(\operatorname{div} ; \Omega):=\{\boldsymbol{v} \in H(\operatorname{div} ; \Omega) \mid \boldsymbol{v} \cdot \boldsymbol{n}=0$ on $\partial \Omega\}$ the variational formulation of Darcy's problem is given by: find $(\boldsymbol{u}, p) \in\left(H_{0}(\operatorname{div} ; \Omega), L_{0}^{2}(\Omega)\right)$ such that for all $(\boldsymbol{v}, q) \in\left(H_{0}(\operatorname{div} ; \Omega), L_{0}^{2}(\Omega)\right)$ we have

$$
\int_{\Omega} \mu \kappa^{-1} \boldsymbol{u} \cdot \boldsymbol{v} \mathrm{d} \boldsymbol{x}-\int_{\Omega} p \nabla \cdot \boldsymbol{v} \mathrm{d} \boldsymbol{x}-\int_{\Omega} q \nabla \cdot \boldsymbol{u} \mathrm{d} \boldsymbol{x}=\int_{\Omega} \boldsymbol{f} \cdot \boldsymbol{v} \mathrm{d} \boldsymbol{x} .
$$

It is well-known (see e.g. [4], Chap. 12, p. 300) that problem (4.8) is equivalent to the following problem: find $\boldsymbol{u}$ in the subspace $H_{0}\left(\operatorname{div}_{0} ; \Omega\right):=\left\{\boldsymbol{v} \in H_{0}(\operatorname{div} ; \Omega) \mid \nabla \cdot \boldsymbol{v} \equiv 0\right\}$ such that

$$
\int_{\Omega} \mu \kappa^{-1} \boldsymbol{u} \cdot \boldsymbol{v} \mathrm{d} \boldsymbol{x}=\int_{\Omega} \boldsymbol{f} \cdot \boldsymbol{v} \mathrm{d} \boldsymbol{x} \quad \forall v \in H_{0}\left(d i v_{0} ; \Omega\right) .
$$

Let us additionally assume that $\Omega$ is simply connected. Then, we have (see e.g. [12]) that there exists exactly one $\phi \in H_{0}^{1}(\Omega)$ such that $\nabla \times \phi=\boldsymbol{u}$, where $\nabla \times \phi:=\left[\frac{\partial \phi}{\partial x_{2}},-\frac{\partial \phi}{\partial x_{1}}\right]$. This leads to the variational form of Darcy's problem in stream function formulation: Find $\phi \in \mathscr{V}_{0}:=H_{0}^{1}(\Omega)$ such that for all $\psi \in \mathscr{V}_{0}$ we have

$$
a^{D}(\phi, \psi):=\int_{\Omega} \mu \kappa^{-1} \nabla \times \phi \cdot \nabla \times \psi \mathrm{d} \boldsymbol{x}=\int_{\Omega} \boldsymbol{f} \cdot(\nabla \times \psi) \mathrm{d} \boldsymbol{x} .
$$

Note that $a^{D}(\phi, \psi)=\int_{\Omega} \mu \kappa^{-1} \nabla \phi \cdot \nabla \psi \mathrm{d} \boldsymbol{x}$. Thus, for $\mathscr{V}_{0}\left(\Omega_{j}\right)$ and $\xi_{j}$ as chosen in Section 4.1 for $j=1, \ldots, n_{\boldsymbol{x}}$ we can readily verify assumptions (A1)-(A4). Let us assume that, as above, $\kappa$ only assumes two values. Then, we can perform exactly the same argument as in Section 4.1 (with $\kappa$ replaced by $\mu \kappa^{-1}$ ) to establish the robustness (with respect to $\kappa_{\max } / \kappa_{\min }$ and $H$ ) of the stable decomposition (3.2) corresponding to the stream function formulation with its bilinear form $a^{D}(\cdot, \cdot)$. Thus, we may robustly precondition (4.9), namely, solve for $\phi$ and recover $\boldsymbol{u}=\nabla \times \phi$ from (4.8).

An equivalent approach, that we use in Section 6 to compute a solution of (4.8), is somewhat different and outlined in the following Remark.

Remark 4.1. Instead of solving the stream function formulation for $\phi$ and then recovering $\boldsymbol{u}=\nabla \times \phi$, one may equivalently use the coarse space corresponding to (4.9) to construct a coarse space corresponding to (4.8) by applying $\nabla \times$ to the coarse stream basis functions. This then yields an equivalent robust additive Schwarz preconditioner for (4.8) (for details see [19], Sect. 10.4.2). 


\subsection{Stokes' equation}

As for the mixed form of the elliptic equation we assume that $\Omega \subset \mathbb{R}^{2}$ is simply connected. Then we consider Stokes' equations modeling slow viscous flows

$$
\left\{\begin{array}{l}
-\mu \Delta \boldsymbol{u}+\nabla p=\boldsymbol{f} \text { in } \Omega \\
\nabla \cdot \boldsymbol{u}=0 \text { in } \Omega \\
\boldsymbol{u}=\mathbf{0} \text { on } \partial \Omega
\end{array}\right.
$$

where $p \in L_{0}^{2}(\Omega), \boldsymbol{u} \in\left(H_{0}^{1}(\Omega)\right)^{2}, \boldsymbol{f} \in\left(L^{2}(\Omega)\right)^{2}$, and $\mu \in \mathbb{R}^{+}$. The variational formulation of the Stokes problem is: find $(\boldsymbol{u}, p) \in\left(\left(H_{0}^{1}(\Omega)\right)^{2}, L_{0}^{2}(\Omega)\right)$ such that for all $(\boldsymbol{v}, q) \in\left(\left(H_{0}^{1}(\Omega)\right)^{2}, L_{0}^{2}(\Omega)\right)$ we have

$$
\int_{\Omega} \mu \nabla \boldsymbol{u}: \nabla \boldsymbol{v} \mathrm{d} \boldsymbol{x}-\int_{\Omega} p \nabla \cdot \boldsymbol{v} \mathrm{d} \boldsymbol{x}-\int_{\Omega} q \nabla \cdot \boldsymbol{u} \mathrm{d} \boldsymbol{x}=\int_{\Omega} \boldsymbol{f} \cdot \boldsymbol{v} \mathrm{d} \boldsymbol{x},
$$

where $\nabla \boldsymbol{u}: \nabla \boldsymbol{v}:=\sum_{i, j=1}^{2} \frac{\partial u_{i}}{\partial x_{j}} \frac{\partial v_{i}}{\partial x_{j}}$ denotes the usual Frobenius product.

Analogously to Section 4.2 we can formulate an equivalent problem for stream functions: Find $\phi \in \mathscr{V}_{0}:=$ $\left\{\psi \in H^{2}(\Omega) \cap H_{0}^{1}(\Omega)\left|\frac{\partial \psi}{\partial \boldsymbol{n}}\right|_{\partial \Omega}=0\right\}$ such that for all $\phi \in \mathscr{V}_{0}$

$$
a^{S}(\phi, \psi):=\int_{\Omega} \mu \nabla(\nabla \times \phi): \nabla(\nabla \times \psi) \mathrm{d} \boldsymbol{x}=\int_{\Omega} \boldsymbol{f} \cdot \nabla \times \psi \mathrm{d} \boldsymbol{x} .
$$

For a sufficiently regular partition of unity $\left\{\xi_{j}\right\}_{j=1}^{n_{x}}$ and spaces $\mathscr{V}_{0}\left(\Omega_{j}\right), j=1, \ldots, n_{\boldsymbol{x}}$, defined as $\mathscr{V}_{0}\left(\Omega_{j}\right):=$ $\left\{\psi \in H^{2}\left(\Omega_{j}\right) \cap H_{0}^{1}\left(\Omega_{j}\right)\left|\frac{\partial \psi}{\partial \boldsymbol{n}}\right|_{\partial \Omega_{j}}=0\right\}$, we can readily verify (A1)-(A4). In order to show that the number of eigenvalues below a suitable threshold is small (independent of $H$ ) we define for $j=1, \ldots, n_{\boldsymbol{x}}$

$$
\mathscr{V}_{S}^{c}\left(\Omega_{j}\right):=\left\{\phi \in \mathscr{V}\left(\Omega_{j}\right) \mid \int_{\Omega_{j}} \phi=0, \int_{\Omega_{j}} \nabla \phi \mathrm{d} \boldsymbol{x}=\mathbf{0}\right\} .
$$

As above, it is straightforward to see that any 4-dimensional subspace has a non-empty intersection with $\mathscr{V}_{S}^{c}\left(\Omega_{j}\right)$. Thus, by again using the min-max principle we see that there exists a $\phi \in \mathscr{V}_{S}^{c}\left(\Omega_{j}\right)$ such that

$$
\lambda_{4}^{j} \geq \frac{a_{\Omega_{j}}^{S}(\phi, \phi)}{m_{\Omega_{j}}^{S}(\phi, \phi)} .
$$

Using the definitions of $a_{\Omega_{j}}^{S}(\cdot, \cdot)$ and $m_{\Omega_{j}}^{S}(\cdot, \cdot)$ (see $(2.5)$ with $a(\cdot, \cdot)$ replaced by $a^{S}(\cdot, \cdot)$ ) we note that

$$
\begin{aligned}
m_{\Omega_{j}}^{S}(\phi, \phi) & =\sum_{i \in I_{j}} a_{\Omega_{j}}^{S}\left(\xi_{j} \xi_{i} \phi, \xi_{j} \xi_{i} \phi\right) \\
& =\sum_{i \in I_{j}} \int_{\Omega_{j}} \mu \mathcal{H}\left(\xi_{j} \xi_{i} \phi\right): \mathcal{H}\left(\xi_{j} \xi_{i} \phi\right) \mathrm{d} \boldsymbol{x} \\
& =\sum_{i \in I_{j}} \int_{\Omega_{j}} \mu\left\|\phi \mathcal{H}\left(\xi_{i} \xi_{j}\right)+\xi_{i} \xi_{j} \mathcal{H}(\phi)+\nabla\left(\xi_{i} \xi_{j}\right) \otimes \nabla \phi+\nabla \phi \otimes \nabla\left(\xi_{i} \xi_{j}\right)\right\|_{\mathcal{F}}^{2} \mathrm{~d} \boldsymbol{x} \\
& \leq C n_{I} \int_{\Omega_{j}} \mu\left(\phi^{2} H^{-4}+\mathcal{H}(\phi): \mathcal{H}(\phi)+H^{-2}(\nabla \phi)^{2}\right) \mathrm{d} \boldsymbol{x},
\end{aligned}
$$

where $C$ only depends on the particular choice of the partition of unity. Here $\mathcal{H}(\phi):=\left[\frac{\partial^{2} \phi}{\partial x_{i} \partial x_{j}}\right]_{i, j=1,2}$ denotes the Hessian of $\phi, \otimes$ the tensor product, and $\|\cdot\|_{\mathcal{F}}$ denotes the Frobenius norm associated with the Frobenius 
product defined above. Since $\phi \in \mathscr{V}_{S}^{c}\left(\Omega_{j}\right)$ we may apply Poincaré's inequality to $\phi$ and its first derivatives. Thus, we obtain

$$
m_{\Omega_{j}}^{S}(\phi, \phi) \leq C n_{I} \int_{\Omega_{j}} \mu \mathcal{H}(\phi): \mathcal{H}(\phi) \mathrm{d} \boldsymbol{x}=C a_{\Omega_{j}}^{S}(\phi, \phi),
$$

where $C$ only depends on the partition of unity and the shape of $\Omega_{j}$ but is independent of $H$. Combining (4.13) with (4.12) we obtain $\lambda_{4}^{j} \geq C$ with $C$ independent of $H$. This establishes that the decomposition (3.2) and the corresponding additive Schwarz preconditioner are robust with respect to $H$. As outlined in Remark 4.1 we can also obtain a robust additive Schwarz preconditioner for (4.10).

\subsection{Brinkman's equation}

As for Darcy's and Stokes' problem, we assume that $\Omega \subset \mathbb{R}^{2}$ is simply connected. Brinkman's problem modeling flows in highly porous media is given by $(c f .[5])$

$$
\left\{\begin{array}{l}
-\mu \Delta \boldsymbol{u}+\nabla p+\mu \kappa^{-1} \boldsymbol{u}=\boldsymbol{f} \text { in } \Omega \\
\nabla \cdot \boldsymbol{u}=0 \text { in } \Omega \\
\boldsymbol{u}=\mathbf{0} \text { on } \partial \Omega
\end{array}\right.
$$

where $p, \boldsymbol{u}, \boldsymbol{f}$, and $\mu$ are chosen as in the Stokes' case and $\kappa$ as in the Darcy case. The variational formulation of the Brinkman problem is: find $(\boldsymbol{u}, p) \in\left(\left(H_{0}^{1}(\Omega)\right)^{2}, L_{0}^{2}(\Omega)\right)$ such that for all $(\boldsymbol{v}, q) \in\left(\left(H_{0}^{1}(\Omega)\right)^{2}, L_{0}^{2}(\Omega)\right)$ we have

$$
\int_{\Omega} \mu \nabla \boldsymbol{u}: \nabla \boldsymbol{v} \mathrm{d} \boldsymbol{x}+\int_{\Omega} \mu \kappa^{-1} \boldsymbol{u} \cdot \boldsymbol{v} \mathrm{d} \boldsymbol{x}-\int_{\Omega} p \nabla \cdot \boldsymbol{v} \mathrm{d} \boldsymbol{x}-\int_{\Omega} q \nabla \cdot \boldsymbol{u} \mathrm{d} \boldsymbol{x}=\int_{\Omega} \boldsymbol{f} \cdot \boldsymbol{v} \mathrm{d} \boldsymbol{x} .
$$

Again, we adopt the setting of stream functions. For $\mathscr{V}_{0}$ as in Section (4.3), the variational stream function formulation reads: Find $\phi \in \mathscr{V}_{0}$ such that for all $\psi \in \mathscr{V}_{0}$ we have

$$
a^{B}(\phi, \psi):=\int_{\Omega} \mu\left(\nabla(\nabla \times \phi): \nabla(\nabla \times \psi)+\kappa^{-1} \nabla \times \phi \cdot \nabla \times \psi\right) \mathrm{d} \boldsymbol{x}=\int_{\Omega} \boldsymbol{f} \cdot \nabla \times \psi \mathrm{d} \boldsymbol{x} .
$$

With $\xi_{j}$ and $\mathscr{V}_{0}\left(\Omega_{j}\right)$ as in Section 4.3 for $j=1, \ldots, n_{\boldsymbol{x}}$ we readily verify (A1)-(A4).

Note that

$$
a^{B}(\phi, \psi)=a^{S}(\phi, \psi)+a^{D}(\phi, \psi) \quad \text { and } \quad m^{B}(\phi, \psi)=m^{S}(\phi, \psi)+m^{D}(\phi, \psi),
$$

where $m^{B}(\cdot, \cdot)$ is defined according to $(2.5)$ with $a(\cdot, \cdot)$ replaced by $a^{B}(\cdot, \cdot)$.

Since for any $\psi \in \mathscr{V}\left(\Omega_{j}\right), j=1, \ldots, n_{\boldsymbol{x}}$, we have $m_{\Omega_{j}}^{S}(\psi, \psi), m_{\Omega_{j}}^{D}(\psi, \psi)>0$ and $a_{\Omega_{j}}^{S}(\psi, \psi), a_{\Omega_{j}}^{D}(\psi, \psi) \geq 0$ we have

$$
\frac{a_{\Omega_{j}}^{B}(\psi, \psi)}{m_{\Omega_{j}}^{B}(\psi, \psi)}=\frac{a_{\Omega_{j}}^{S}(\psi, \psi)+a_{\Omega_{j}}^{D}(\psi, \psi)}{m_{\Omega_{j}}^{S}(\psi, \psi)+m_{\Omega_{j}}^{D}(\psi, \psi)} \geq \min \left\{\frac{a_{\Omega_{j}}^{S}(\psi, \psi)}{m_{\Omega_{j}}^{S}(\psi, \psi)}, \frac{a_{\Omega_{j}}^{D}(\psi, \psi)}{m_{\Omega_{j}}^{D}(\psi, \psi)}\right\} .
$$

This is an immediate consequence of the following inequality, valid for $\beta_{1}, \beta_{2} \geq 0$ and $\beta_{3}, \beta_{4}>0$

$$
\frac{\beta_{1}+\beta_{2}}{\beta_{3}+\beta_{4}}=\frac{\frac{\beta_{1}}{\beta_{3} \beta_{4}}+\frac{\beta_{2}}{\beta_{3} \beta_{4}}}{\frac{1}{\beta_{4}}+\frac{1}{\beta_{3}}} \geq\left\{\begin{array}{ll}
\frac{\beta_{2}}{\beta_{4}}, & \text { if } \frac{\beta_{1}}{\beta_{3}} \geq \frac{\beta_{2}}{\beta_{4}} \\
\frac{\beta_{1}}{\beta_{3}}, & \text { if } \frac{\beta_{2}}{\beta_{4}} \geq \frac{\beta_{1}}{\beta_{3}}
\end{array}\right\} \geq \min \left\{\frac{\beta_{1}}{\beta_{3}}, \frac{\beta_{2}}{\beta_{4}}\right\} .
$$

Combining (4.17) with the results from Sections 4.2 and 4.3 we obtain

$$
\lambda_{\max \left\{L_{j}+1,4\right\}}^{j} \geq C,
$$

where $C$ is independent of $H$ and $\kappa_{\max } / \kappa_{\min }$, and where $L_{j}$ is chosen as in Section 4.2. Thus we establish that the decomposition introduced by (3.2) and the corresponding additive Schwarz preconditioner are robust with respect to $H$ and $\kappa_{\max } / \kappa_{\min }$. As for the Darcy and the Stokes case, we can also obtain an equivalent robust additive Schwarz preconditioner for (4.15) (see Rem. 4.1). 

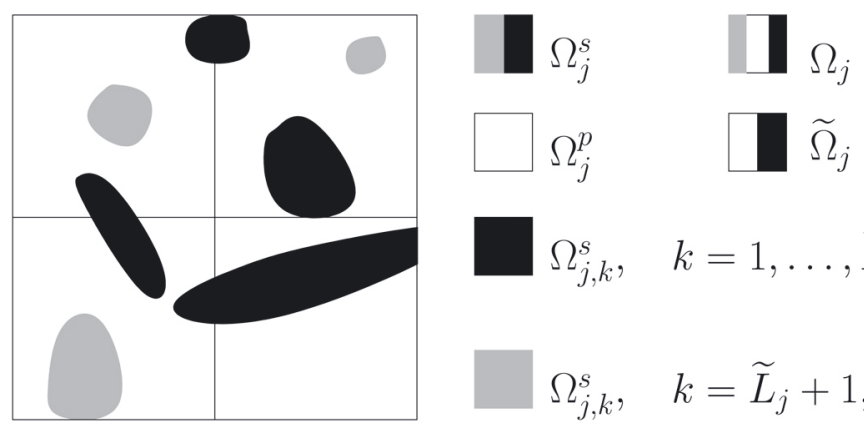

$$
k=1, \ldots, \widetilde{L}_{j}
$$$$
k=\widetilde{L}_{j}+1, \ldots, L_{j}
$$

FIGURE 3. Subdomain with connected components of $\Omega_{j}^{s}$. The black connected components are those touching an edge interior to $\Omega_{j}$. The gray connected components are the remaining ones. In this configuration $\widetilde{L}_{j}=4$ and $L_{j}=7$.

\section{REDUCING THE DIMENSION OF THE COARSE SPACE}

In the exposition above, only assumption (A4) was made about the choice of the partition of unity $\left\{\xi_{j}\right\}_{j=1}^{n_{x}}$. In this section, we investigate possibilities of making a particular choice of the partition of unity denoted by $\left\{\widetilde{\xi}_{j}\right\}_{j=1}^{n_{x}}$ that results in a reduction of the dimension of the coarse space $\mathscr{V}_{H}$. As indicated in Remark 3.1 we do this in the setting of infinite dimensional function spaces. The idea is that by replacing $\left\{\xi_{j}\right\}_{j=1}^{n_{x}}$ with $\left\{\widetilde{\xi}_{j}\right\}_{j=1}^{n_{x}}$ one can avoid the asymptotically small eigenvalues for those connected components of $\Omega^{s}$ which do not touch the boundary of any coarse cell $T \in \mathcal{T}_{H}$. For this, we again assume the scalar elliptic setting as in Section 4.1.

Let $\left\{\xi_{j}\right\}_{j=1}^{n_{\boldsymbol{x}}}$ be a standard partition of unity as above. For each $j=1, \ldots, n_{\boldsymbol{x}}$ and each $T \subset \Omega_{j}$, let $\left.\widetilde{\xi}_{j}\right|_{T}$ be the solution of

$$
-\nabla \cdot\left(\kappa \nabla \widetilde{\xi}_{j}\right)=0, \quad \text { in } T, \quad \widetilde{\xi}_{j}=\xi_{j}, \quad \text { on } \partial T .
$$

The corresponding variational formulation reads: Find $\left.\widetilde{\xi}_{j}\right|_{T} \in H_{0}^{1}(T)+\xi_{j}$ such that for all $\psi \in H_{0}^{1}(T)$ we have

$$
a_{T}^{S E}\left(\widetilde{\xi}_{j}, \psi\right)=0, \quad \forall \psi \in H_{0}^{1}(T) .
$$

We set $\widetilde{\xi}_{j} \equiv 0$ in $\Omega \backslash \Omega_{j}$ and check whether with $\left\{\widetilde{\xi}_{j}\right\}_{j=1}^{n_{x}}$ instead of $\left\{\xi_{j}\right\}_{j=1}^{n_{x}}$ (A4) is satisfied.

As in Section 4.1 let $\Omega_{j, k}^{s}, k=1, \ldots, L_{j}$ be the $k$-th connected component of $\Omega_{j}^{s}$. Without loss of generality we may assume a numbering such that $\Omega_{j, k}^{s}$ for $k=1, \ldots, \widetilde{L}_{j}\left(\leq L_{j}\right)$ is a connected component for which $\bar{\Omega}_{j, k}^{s} \cap\left(\partial T \backslash \partial \Omega_{j}\right) \neq \emptyset$ for some $T \subset \Omega_{j}$. Note, that in general $\widetilde{L}_{j} \leq L_{j}$ and that $L_{j}-\widetilde{L}_{j}$ is precisely the number of connected components of $\Omega_{j}^{s}$ which do not touch an edge of $T \subset \Omega_{j}$ interior to $\Omega_{j}$, i.e., for $i=\widetilde{L}_{j}+1, \ldots, L_{j}$ we have that $\bar{\Omega}_{j, i}^{s} \cap\left(\partial T \backslash \partial \Omega_{j}\right)=\emptyset$. We also define $\widetilde{\Omega}_{j}:=\Omega_{j} \backslash\left(\bigcup_{k=\widetilde{L}_{j}+1}^{L_{j}} \bar{\Omega}_{j, k}^{s}\right)$. These notations are illustrated in

Figure 3.

To proceed we make the following assumption:

$(\widetilde{\mathrm{A}})$ For $\widetilde{\xi}_{j}$ defined as above, we have

$$
\left\|\nabla \widetilde{\xi}_{j}\right\|_{L^{\infty}\left(\Omega_{j}\right)} \leq C H^{-1} \quad \text { and } \quad\left\|\kappa_{\max } \nabla \widetilde{\xi}_{j}\right\|_{L^{\infty}\left(\Omega_{j} \backslash \widetilde{\Omega}_{j}\right)} \leq C H^{-1}, j=1, \ldots, n_{\boldsymbol{x}}
$$

where $C$ is independent of $\kappa_{\max } / \kappa_{\min }$ and $H$. 
Note that by [9], Lemma 3.1, $\left\|\nabla \widetilde{\xi}_{j}\right\|_{L^{2}\left(\Omega_{j}\right)}^{2} \leq C H^{n-2}$ and that by essentially the same argument as in [13], Theorems 4.3 and 4.5 , we have that $\left\|\kappa_{\max } \nabla \widetilde{\xi}_{j}\right\|_{L^{2}\left(\Omega_{j} \backslash \widetilde{\Omega}_{j}\right)}^{2} \leq C H^{n-2}$, where as above $n$ denotes the spatial dimension. To the best of our knowledge, obtaining rigorous $L^{\infty}$-estimates as stated in $(\widetilde{\mathrm{A}})$ is still an open problem and beyond the scope of this paper.

It is easy to see that $\sum_{j=1}^{n_{\boldsymbol{x}}} \widetilde{\xi}_{j} \equiv 1$ and that $\operatorname{supp}\left(\widetilde{\xi}_{j}\right)=\bar{\Omega}_{j}$ for any $j=1, \ldots, n_{\boldsymbol{x}}$. Thus, to establish the validity of (A4) it remains to verify that $\widetilde{\xi}_{j} \psi \in \mathscr{V}_{0}=H_{0}^{1}(\Omega)$ and $\widetilde{\xi}_{j} \psi \in \mathscr{V}_{0}\left(\Omega_{j}\right)$ for all $\psi \in \mathscr{V}_{0}$. For this we restrict to the case of two spatial dimensions, i.e., $n=2$ :

Note that for some $\epsilon>0$ we have that $\widetilde{\xi}_{j} \in H^{1+\epsilon}\left(\Omega_{j}\right)$ (cf. [14]). Thus, by [1], Theorem 7.57, we know that $\widetilde{\xi}_{j} \in L^{\infty}\left(\Omega_{j}\right)$. Using this and $(\widetilde{\mathrm{A}})$ we see that

$$
\begin{aligned}
\left\|\nabla\left(\widetilde{\xi}_{j} \psi\right)\right\|_{L^{2}\left(\Omega_{j}\right)} & \leq\left\|\widetilde{\xi}_{j} \nabla \psi\right\|_{L^{2}\left(\Omega_{j}\right)}+\left\|\psi \nabla \widetilde{\xi}_{j}\right\|_{L^{2}\left(\Omega_{j}\right)} \\
& \leq C\left(\|\nabla \psi\|_{L^{2}\left(\Omega_{j}\right)}+H^{-1}\|\psi\|_{L^{2}\left(\Omega_{j}\right)}\right)<\infty .
\end{aligned}
$$

It is furthermore easy to see that $\operatorname{supp}\left(\widetilde{\xi}_{j} \psi\right) \subset \bar{\Omega}_{j}$, which establishes (A4).

Similarly to Section 4.1 we now define

$$
\widetilde{\mathscr{V}}_{S E}^{c}\left(\Omega_{j}\right):=\left\{\phi \in \mathscr{V}\left(\Omega_{j}\right) \mid \int_{\Omega_{j, k}^{s}} \phi \mathrm{d} \boldsymbol{x}=0, \text { for } k=1, \ldots, \widetilde{L}_{j}\right\},
$$

and by the min-max principle we know that there exists a $\phi \in \widetilde{\mathscr{V}}_{S E}^{c}\left(\Omega_{j}\right)$ such that

$$
\widetilde{\lambda}_{\widetilde{L}_{j}+1}^{j} \geq \frac{a_{\Omega_{j}}^{S E}(\phi, \phi)}{\widetilde{m}_{\Omega_{j}}^{S E}(\phi, \phi)}
$$

where $\widetilde{m}_{\Omega_{j}}^{S E}(\cdot, \cdot)$ is defined as $m_{\Omega_{j}}^{S E}(\cdot, \cdot)$ with $\left\{\xi_{j}\right\}_{j=1}^{n_{x}}$ replaced by $\left\{\widetilde{\xi}_{j}\right\}_{j=1}^{n_{x}}$.

In order to obtain a uniform (with respect to $\kappa_{\max } / \kappa_{\min }$ and $H$ ) lower bound for $\widetilde{\lambda}_{\widetilde{L}_{j}+1}^{j}$, we need to verify that

$$
\widetilde{m}_{\Omega_{j}}^{S E}(\phi, \phi) \leq C a_{\Omega_{j}}^{S E}(\phi, \phi),
$$

with $C$ independent of $\kappa_{\max } / \kappa_{\min }$ and $H$.

Revisiting estimate (4.5), we obtain

$$
\widetilde{m}_{\Omega_{j}}^{S E}(\phi, \phi) \leq 2 \sum_{i \in I_{j}} \int_{\Omega_{j}} \kappa\left|\nabla\left(\widetilde{\xi}_{j} \widetilde{\xi}_{i}\right) \phi\right|^{2} \mathrm{~d} \boldsymbol{x}+2 a_{\Omega_{j}}^{S E}(\phi, \phi) .
$$

Thus, it suffices to bound for any $i \in I_{j}$

$$
\int_{\Omega_{j}} \kappa\left|\nabla\left(\widetilde{\xi}_{j} \widetilde{\xi}_{i}\right) \phi\right|^{2} \mathrm{~d} \boldsymbol{x}=\underbrace{\int_{\widetilde{\Omega}_{j}} \kappa\left|\nabla\left(\widetilde{\xi}_{j} \widetilde{\xi}_{i}\right) \phi\right|^{2} \mathrm{~d} \boldsymbol{x}}_{=: E_{4}}+\underbrace{\int_{\Omega_{j} \backslash \widetilde{\Omega}_{j}} \kappa\left|\nabla\left(\widetilde{\xi}_{j} \widetilde{\xi}_{i}\right) \phi\right|^{2} \mathrm{~d} \boldsymbol{x}}_{=: E_{5}}
$$

by $a_{\Omega_{j}}^{S E}(\phi, \phi)$. To avoid unnecessary technicalities, we make the simplifying assumption that each connected component of $\widetilde{\Omega}_{j}$ contains at least one $\Omega_{j, k}^{s}$ with $k=1, \ldots, \widetilde{L}_{j}$. If this assumption is violated, one simply needs 
to introduce additional conditions in (5.2) ensuring that the average of functions is zero over each connected component of $\widetilde{\Omega}_{j}$ that does not contain any $\Omega_{j, k}^{s}$ with $k=1, \ldots, \widetilde{L}_{j}$.

Assuming $(\widetilde{\mathrm{A}})$, we can find the required estimate of $E_{4}$ as follows:

$$
E_{4} \leq C H^{-2} \int_{\widetilde{\Omega}_{j}} \kappa \phi^{2} \mathrm{~d} \boldsymbol{x} \leq C \int_{\widetilde{\Omega}_{j}} \kappa|\nabla \phi|^{2} \mathrm{~d} \boldsymbol{x} \leq C a_{\Omega_{j}}^{S E}(\phi, \phi),
$$

where the second inequality follows by a reasoning analogous to (4.6), which is possible since $\phi \in \widetilde{\mathcal{V}}_{S E}^{c}\left(\Omega_{j}\right)$. Due to $(\widetilde{\mathrm{A}}), C$ can be chosen independently of $\kappa_{\max } / \kappa_{\min }$ and $H$, but it may depend on the geometry of $\widetilde{\Omega}_{j}$.

For an estimate of $E_{5}$ note that by $(\widetilde{\mathrm{A}})$

$$
\begin{aligned}
E_{5} & \leq 2 \kappa_{\max } \int_{\Omega_{j} \backslash \tilde{\Omega}_{j}}\left(\left|\nabla \widetilde{\xi}_{j}\right|^{2}+\left|\nabla \widetilde{\xi}_{i}\right|^{2}\right) \phi^{2} \mathrm{~d} \boldsymbol{x} \\
& \leq C H^{-2} \int_{\Omega_{j} \backslash \tilde{\Omega}_{j}} \phi^{2} \mathrm{~d} \boldsymbol{x} \leq C \int_{\Omega_{j}}|\nabla \phi|^{2} \mathrm{~d} \boldsymbol{x} \leq C a_{\Omega_{j}}^{S E}(\phi, \phi),
\end{aligned}
$$

where we have used Poincaré's inequality. This establishes (5.4), which yields that $\widetilde{\lambda}_{\widetilde{L}_{j}+1}^{j}$ in (5.3) is bounded below independently of the contrast and $H$.

In analogy to (3.1) we define the coarse space, called further multiscale spectral coarse space, that is constructed using $\left\{\widetilde{\xi}_{j}\right\}_{j=1}^{n_{x}}$ instead of $\left\{\xi_{j}\right\}_{j=1}^{n_{x}}$ by

$$
\widetilde{\mathscr{V}}_{H}:=\operatorname{span}\left\{\widetilde{\xi}_{j} \widetilde{\varphi}_{i}^{j} \mid j=1, \ldots, n_{\boldsymbol{x}} \text { and } i=1, \ldots, \widetilde{L}_{j}\right\},
$$

where $\widetilde{\varphi}_{i}^{j}$ are given by $(2.6)$ with $m_{\Omega_{j}}(\cdot, \cdot)$ replaced by $\widetilde{m}_{\Omega_{j}}^{S E}(\cdot, \cdot)$.

Remark 5.1. We note that for any subdomain $\Omega_{j}$ with $\bar{\Omega}_{j} \cap \partial \Omega=\emptyset$, we have that $\left(0, \mathbf{1}_{\Omega_{j}}\right)$ is an eigenpair of the generalized eigenvalue problem posed on $\Omega_{j}$, where $\mathbf{1}_{\Omega_{j}}$ denotes the constant 1-function on $\Omega_{j}$. Thus, all multiscale partition of unity functions $\widetilde{\xi}_{j}$ corresponding to subdomains $\Omega_{j}$ with $\bar{\Omega}_{j} \cap \partial \Omega=\emptyset$ are basis functions of our coarse space $\widetilde{\mathscr{V}}_{H}$. This observation allows the interpretation of our method as a procedure that enriches a multiscale coarse space (given by the span of the multiscale partition of unity functions) by features that cannot be represented locally. These features are incorporated by those eigenfunctions corresponding to non-zero (but small) eigenvalues.

\section{Numerical RESUlts}

\subsection{General setting}

In this section, we investigate the performance of the overlapping Schwarz domain decomposition method with coarse spaces discussed above when applied to some specific problems described in Section 4. We have implemented this preconditioner in $\mathrm{C}++$ using the finite element library deal.II (cf. [2]). Our goal is to experimentally establish the robustness of additive Schwarz preconditioners with respect to contrast $\kappa_{\max } / \kappa_{\min }$. The comparison is made using some coarse spaces known in the literature and the coarse spaces introduced in this paper. Namely, we consider the following coarse spaces.

1. Standard coarse space, denoted by $\mathscr{V}_{H}^{s t}:=\operatorname{span}\left\{\xi_{j}\left|\quad \xi_{j}\right|_{\partial \Omega_{j}} \equiv 0\right.$ for $\left.j=1, \ldots, n_{\boldsymbol{x}}\right\}$, of standard partition of unity functions corresponding to interior coarse mesh nodes, which were introduced above (see also, e.g. [27]);

2. multiscale coarse space, denoted by $\mathscr{V}_{H}^{m s}:=\operatorname{span}\left\{\widetilde{\xi}_{j}\left|\widetilde{\xi}_{j}\right|_{\partial \Omega_{j}} \equiv 0\right.$ for $\left.j=1, \ldots, n_{\boldsymbol{x}}\right\}$, of functions that over each $\Omega_{j}$ are solutions of problem (5.1) and correspond to interior coarse mesh nodes (cf. [13]);

3. spectral coarse space, defined in (3.1) as $\mathscr{V}_{H}:=\operatorname{span}\left\{\xi_{j} \varphi_{i}^{j} \mid j=1, \ldots, n_{\boldsymbol{x}}\right.$ and $\left.i=1, \ldots, L_{j}\right\}$;

4. multiscale spectral coarse space, defined in (5.5) (used only in the scalar elliptic case, see Sect. 5). 

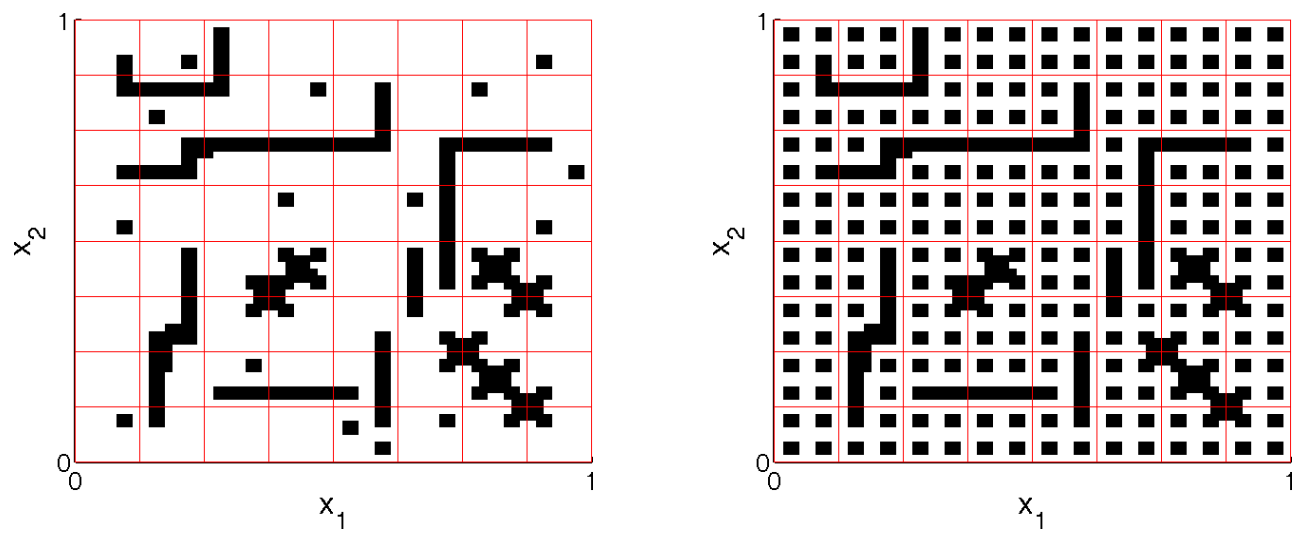

FiguRE 4. Two sample geometries: geometry 1 (left) and geometry 2 (right); the regions of low (white) and high (black) values of the coefficients. The mesh indicates the coarse triangulation.

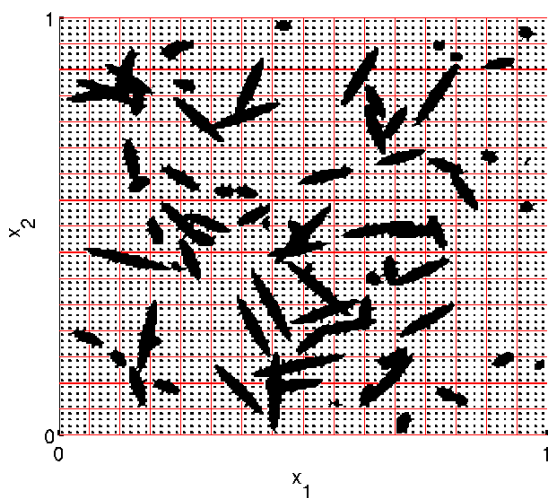

(a) Geometry 3: periodic background and randomly distributed inclusions.

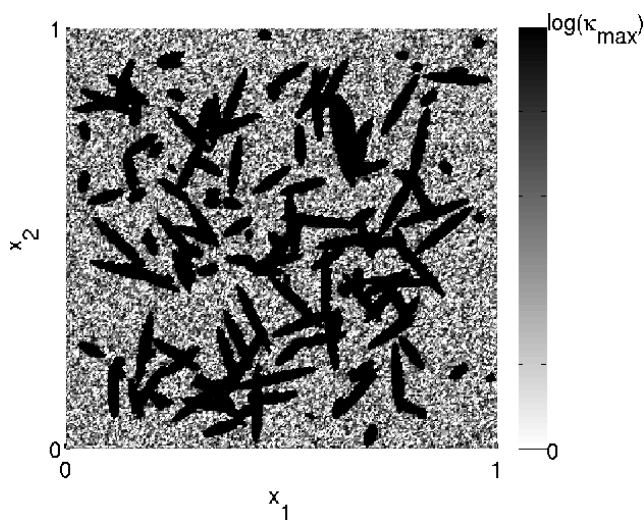

(b) Geometry 4: random background and randomly distributed inclusions - logarithmic plot of $\kappa$.

Figure 5. Two geometries with a $256 \times 256$ fine mesh and a $16 \times 16$ coarse mesh.

In all numerical examples (unless stated otherwise), the threshold for taking into account eigenpairs for the construction of the coarse space is chosen to be 0.5 , i.e., $1 / \tau_{\lambda}=0.5$. In practice one is generally interested in choosing $1 / \tau_{\lambda}$ not too large to avoid an unnecessarily large dimension of the coarse space. We recall that, in general, in order to obtain methods that are robust with respect to the contrast, one needs to include all eigenvectors corresponding to contrast-dependent small eigenvalues into the construction of the coarse space. For the cases with a clear gap between contrast-dependent eigenvalues and the rest of the spectrum we can easily select a threshold $1 / \tau_{\lambda}$. These cases include for example, very high-contrast coefficient with isolated highconductivity channels and inclusions. For cases with no clear gap in the spectrum (i.e., continuous range of conductivity values) a fixed threshold should be used for the construction of coarse spaces.

We consider geometries $1-4$ (see Figs. 4 and 5 ), where for geometries $1-3 \kappa$ is equal to $\kappa_{\min }$ and $\kappa_{\text {max }}$ in the white and black regions, respectively, and for geometry $4 \kappa$ is given as shown in the logarithmic plot of Figure 5b.

The geometries shown in Figure 4 differ by the number of connected subregions with high permeability. The goal for these two different distributions of the high contrast is to (1) test the robustness of the developed 
TABLE 1. Elliptic problem of second order: numerical results for standard and spectral coarse spaces.

\begin{tabular}{|c|c|c|c|c|c|c|}
\cline { 2 - 7 } \multicolumn{1}{c|}{} & \multicolumn{3}{c|}{ Standard coarse space $\mathscr{V}_{H}^{s t}$} & \multicolumn{3}{c|}{ Spectral coarse space $\mathscr{V}_{H}$} \\
\hline$\kappa_{\text {max }} / \kappa_{\text {min }}$ & $\#$ iter. & $\operatorname{dim} \mathscr{V}_{H}^{s t}$ & cond. num. & \# iter. & dim. $\mathscr{V}_{H}$ & cond. num. \\
\hline $1 e 2$ & 29 & 49 & $2.29 \mathrm{e} 1$ & 25 & 76 & 15.59 \\
\hline $1 e 3$ & 50 & 49 & $1.88 \mathrm{e} 2$ & 21 & 145 & 11.51 \\
\hline $1 e 4$ & 55 & 49 & $1.79 \mathrm{e} 3$ & 18 & 162 & 6.20 \\
\hline $1 e 5$ & 67 & 49 & $1.78 \mathrm{e} 4$ & 18 & 162 & 6.18 \\
\hline $1 e 6$ & 66 & 49 & $1.77 \mathrm{e} 5$ & 19 & 162 & 6.19 \\
\hline
\end{tabular}

preconditioners with respect to the contrast and (2) show the benefits of the multiscale coarse space in the case of a large number of not connected, isolated, inclusions with high conductivity.

We use a fine grid which is obtained from the coarse grid $\mathcal{T}_{H}$ by subdividing the coarse grid elements into a number of finer elements. For geometry 1 and 2 , we make an initial $8 \times 8$ mesh and introduce in each rectangular element an $8 \times 8$ fine mesh, denoted by $\mathcal{T}_{h}$. Then, the spaces $\mathscr{V}_{0}, \mathscr{V}_{0}\left(\Omega_{j}\right)$ and $\mathscr{V}\left(\Omega_{j}\right)$ are finite element spaces corresponding to this mesh for a specific finite element, which needs to be chosen appropriately for the problem under investigation, e.g, Lagrange finite elements for the scalar elliptic problem in Galerkin formulation. In order not to overburden the notations, we have omitted the dependence of the spaces upon the fine-grid mesh size hoping that this will not lead to a confusion.

\subsection{Numerical experiments for geometry 1}

In this section we use the standard coarse space $\mathscr{V}_{H}^{s t}$ and the spectral coarse space $\mathscr{V}_{H}$, defined in (3.1).

\subsubsection{Scalar elliptic problem - Galerkin formulation (see Sect. 4.1)}

Here, the finite element space is the space of Lagrange finite elements of degree 1 . The right hand side $f$ in (4.1) is chosen to compensate for the boundary condition of linear temperature drop in $x$-direction, i.e., $\phi(\boldsymbol{x})=1-x_{1}$ on $\partial \Omega$. More precisely, we choose $f=\nabla \cdot(\kappa \nabla \widetilde{\phi})$ with $\widetilde{\phi}=1-x_{1}$ in $\Omega$ and where $\nabla \cdot$ is computed in the sense of distributions. Computing then a solution $\widehat{\phi}$ of (4.1) with homogeneous boundary conditions and right hand side $f$, we obtain $\phi=\widehat{\phi}+\widetilde{\phi}$ solving (4.1) with the desired (inhomogeneous) linear temperature drop boundary conditions and homogeneous right hand side.

The dimension of the fine-grid space is 4225 . In the preconditioned conjugate gradient (PCG) method we iterate to achieve a relative reduction of the preconditioned residual of $1 e-6$. In Tables 1 and 2 we present the results of two kinds of numerical experiments on the problem described in Section 4.1 for geometry 1 with contrast $\kappa_{\max } / \kappa_{\min }$ increasing from $1 e 2$ to $1 e 6$. As partition of unity $\left\{\xi_{j}\right\}_{j=1}^{n_{x}}$, we use Lagrange finite element functions of degree 1 corresponding to the coarse mesh $\mathcal{T}_{H}$.

In Table 1 we compare the number of PCG-iterations and the condition numbers for two preconditioners based on the standard coarse space $\mathscr{V}_{H}^{s t}$ (consisting only of the coarse Lagrange finite element functions) and the spectral coarse spaces $\widetilde{\mathscr{V}}_{H}$ generated by our method, respectively. The standard coarse space has fixed dimension 49. The method performs well for low contrasts, but the condition number of the preconditioned systems as well as the number of iterations grow with increasing contrast. The spectral coarse space keeps the condition number independent of the contrast, which is in agreement with our theory.

It seems that the results in the number of iterations for the space $\mathscr{V}_{H}^{s t}$ in the last row in Table 1 deviates from the general trend. We note that for all cases we run the PCG-method with the same stopping criterion, i.e., reduction of the initial preconditioned residual by a factor of $1 e-6$. However, in this case the condition number of the preconditioned system is $1.77 e 5$. Therefore, after reducing the initial preconditioned residual by 
TABLE 2. Elliptic problem of second order: numerical results for spectral coarse spaces $\mathscr{V}_{H}$ of fixed dimension and fixed threshold $1 / \tau_{\lambda}=0.5$.

\begin{tabular}{|c|c|c|c|c|c|c|}
\cline { 2 - 7 } \multicolumn{1}{c|}{} & \multicolumn{3}{c|}{ Spectral coarse space of $\operatorname{dim} \mathscr{V}_{H}=162$} & \multicolumn{3}{c|}{ Spectral coarse space, $1 / \tau_{\lambda}=0.5$} \\
\hline$\kappa_{\max } / \kappa_{\min }$ & \# iter. & cond. & $1 / \tau_{\lambda}$ & \# iter & cond. & $\operatorname{dim} \mathscr{V}_{H}$ \\
\hline $1 e 2$ & 18 & 7.45 & 1.39 & 25 & 15.6 & 76 \\
\hline $1 e 3$ & 17 & 5.99 & 0.92 & 21 & 11.5 & 145 \\
\hline $1 e 4$ & 18 & 6.20 & 0.50 & 18 & 6.20 & 162 \\
\hline $1 e 5$ & 18 & 6.18 & 0.50 & 18 & 6.18 & 162 \\
\hline $1 e 6$ & 19 & 6.19 & 0.50 & 19 & 6.19 & 162 \\
\hline
\end{tabular}

a factor of $1 e-6$ we may still be far away from the solution. Apparently, for larger condition numbers we may need many more iterations to compute the solution accurately.

In Table 2 we show the number of PCG-iterations and condition numbers for two preconditioners based on spectral coarse spaces. In columns 2-4 we report the results for a coarse space of fixed dimension 162 and the threshold for which this is achieved. In columns $5-7$ we present the results for a fixed threshold $1 / \tau_{\lambda}=0.5$. We note that the difference in the performance is only for values of the contrast below $1 e 4$.

\subsubsection{Scalar elliptic problem - mixed formulation (see Sect. 4.2)}

Here, the finite element space is the Raviart-Thomas space of degree 0 ( $R T 0)$ for the velocity and piecewise constants for the pressure on the same rectangular fine mesh as above. Similarly to Section 6.2.1, the right hand side $\boldsymbol{f}$ in (4.7) is chosen to compensate for the boundary condition of unit flow in $x$-direction, i.e., $\boldsymbol{u} \cdot \boldsymbol{n}=\boldsymbol{e}_{1} \cdot \boldsymbol{n}$ on $\partial \Omega$, where $\boldsymbol{e}_{1}$ is the first Cartesian unit vector. The (divergence free) coarse velocity space is constructed as outlined in Remark 4.1. We first construct a basis of the spectral coarse space corresponding to the stream function space. The corresponding coarse velocity space is then given by the span of the curl of these basis functions. Note, that the stream function space corresponding to $R T 0$ is given by the space of Lagrange polynomials of degree 1 (see [12], Sect. 4.4).

As partition of unity $\left\{\xi_{j}\right\}_{j=1}^{n_{x}}$ we could simply use the bilinear Lagrange basis functions corresponding to the coarse mesh $\mathcal{T}_{H}$. Nevertheless, for consistency with the Brinkman case (see Sect. 6.2.3), where we have higher regularity requirements, we choose the $\xi_{j}$ 's to be piecewise polynomials of degree 3 , such that all first derivatives and the lowest mixed derivatives are continuous and $\xi_{j}\left(\boldsymbol{x}_{i}\right)=\delta_{i, j}$ for $i, j=1, \ldots, n_{\boldsymbol{x}}$.

In Table 3 we present the numerical results for this problem and geometry 1 (see Fig. 4). The dimension of the fine space is 12416 . In columns $2-4$ we report the number of iterations, the size of the standard coarse space, and the condition number of the preconditioned system. Here, the standard coarse (velocity) space is given by the span of the curl of the partition of unity functions corresponding to interior coarse mesh nodes. Columns $5-7$ contain the number of iterations, the dimension of the coarse space, as well as the condition number of the preconditioned system. It is clear that for the standard coarse space of dimension 49 the condition number grows with increasing the contrast and so does the number of iterations. However, when the coarse space includes all coarse eigenfunctions below the threshold $1 / \tau_{\lambda}=0.5$, the preconditioner shows convergence rates and condition numbers independent of the contrast.

\subsubsection{Brinkman problem (see Sect. 4.4)}

Next, we present the numerical experiments for the Brinkman problem (4.14). Similarly to Section 6.2.1 the right hand side $\boldsymbol{f}$ is chosen to compensate for the boundary condition of unit flow in $x$-direction, i.e., $\boldsymbol{u}=\boldsymbol{e}_{1}$ on $\partial \Omega$. The viscosity $\mu$ is chosen to be 0.01 and $\kappa$ varies depending on the contrast (see Tab. 4 ).

We discretize this problem with an $H(d i v)$-conforming discontinuous Galerkin discretization (cf. [30,31]) using Raviart-Thomas finite elements of degree 1 (RT1). We again employ a $64 \times 64$ fine grid. It is well-known 
TABLE 3. Scalar elliptic equation in mixed formulation: numerical results for Standard coarse space $\mathscr{V}_{H}^{s t}$ and spectral coarse spaces $\mathscr{V}_{H}$ obtained for a fixed threshold $1 / \tau_{\lambda}=0.5$.

\begin{tabular}{|c|c|c|c|c|c|c|}
\cline { 2 - 7 } \multicolumn{1}{c|}{} & \multicolumn{3}{c|}{ Standard coarse space $\mathscr{V}_{H}^{\text {st }}$} & \multicolumn{3}{c|}{ Spectral coarse space $\mathscr{V}_{H}$} \\
\hline$\kappa_{\text {max }} / \kappa_{\text {min }}$ & \# iter. & $\operatorname{dim} \mathscr{V}_{H}^{s t}$ & cond. num. & \# iter. & $\operatorname{dim} \mathscr{V}_{H}$ & cond. num. \\
\hline $1 e 2$ & 32 & 49 & $2.87 \mathrm{e} 1$ & 23 & 86 & 13.87 \\
\hline $1 e 3$ & 50 & 49 & $2.26 \mathrm{e} 2$ & 24 & 129 & 18.38 \\
\hline $1 e 4$ & 63 & 49 & $2.19 \mathrm{e} 3$ & 17 & 162 & 6.57 \\
\hline $1 e 5$ & 80 & 49 & $2.18 \mathrm{e} 4$ & 18 & 162 & 6.65 \\
\hline $1 e 6$ & 87 & 49 & $2.13 \mathrm{e} 5$ & 19 & 162 & 6.68 \\
\hline
\end{tabular}

TABLE 4. Numerical results for Brinkman's equation using standard coarse space $\mathscr{V}_{H}^{s t}$ and spectral coarse spaces $\mathscr{V}_{H}$.

\begin{tabular}{|c|c|c|c|c|c|c|}
\cline { 2 - 7 } \multicolumn{1}{c|}{} & \multicolumn{3}{c|}{ Standard coarse space $\mathscr{V}_{H}^{s t}$} & \multicolumn{3}{c|}{ spectral coarse space $\mathscr{V}_{H}$} \\
\hline$\kappa_{\text {max }} / \kappa_{\text {min }}$ & $\#$ iter. & dim. $\mathscr{V}_{H}^{s t}$ & cond. num. & \# iter. & dim. $\mathscr{V}_{H}$ & cond. num. \\
\hline $1 e 2$ & 27 & 49 & $2.13 \mathrm{e} 1$ & 25 & 60 & 14.69 \\
\hline $1 e 3$ & 39 & 49 & $4.22 \mathrm{e} 2$ & 28 & 75 & 21.73 \\
\hline $1 e 4$ & 70 & 49 & $2.25 \mathrm{e} 3$ & 29 & 106 & 21.83 \\
\hline $1 e 5$ & 91 & 49 & $1.51 \mathrm{e} 4$ & 24 & 153 & 13.08 \\
\hline $1 e 6$ & 113 & 49 & $1.24 \mathrm{e} 5$ & 22 & 164 & 13.82 \\
\hline
\end{tabular}

(see [12], Sect. 4.4) that in two spatial dimensions the stream function space corresponding to the RT1 space is given by Lagrange biquadratic finite elements. For a generalization to three spatial dimensions one has to utilize Nédélec elements of appropriate degree. As above, we use an $8 \times 8$ coarse mesh. We choose $\left\{\xi_{j}\right\}_{j=1}^{n_{x}}$ as described in Section 6.2.2, which satisfies all regularity constraints.

In Table 4 we give the number of iterations, the dimension of the coarse space in the additive Schwarz preconditioner, as well as the estimated condition number of the preconditioned system. The dimension of the fine space is 49408 . As for the scalar elliptic case in mixed formulation, the coarse (divergence free) velocity space is constructed as outlined in Remark 4.1. In columns $2-5$ we present the results for the case of the standard coarse space of dimension 49, which as in Section 6.2.2 is given by the span of the curl of the partition of unity functions corresponding to interior coarse mesh nodes.

We observe, that the increase in the contrast leads to an increase in the condition number and subsequently to an increase of the number of iterations. Further, in columns 5-7 we report the number of iterations, the dimension of the coarse space in the additive Schwarz preconditioner, as well as the estimated condition number of the preconditioned system for the spectral coarse space obtained by a fixed threshold $1 / \tau_{\lambda}=0.5$. For the Brinkman problem the performance of the preconditioner is also robust. We should note however, that Brinkman's equation is much more difficult to solve due to the fact that the overall system of linear equations is a saddle point problem.

\subsection{Numerical experiments for geometry 2 in Figure 4}

These numerical experiments are aimed to compare the performance of the iterative method applied to the second order elliptic problem in Galerkin formulation (see Sect. 4.1) for a permeability given in geometry 2 (see Fig. 4). The boundary conditions and $f$ are chosen as in Section 6.2.1. The goal here is to demonstrate the coarse space dimension reduction when using multiscale partition of unity functions instead of standard ones. The dimension of the fine-grid space is 4225 . 
TABLE 5. Scalar elliptic - Galerkin formulation: results for spectral coarse space $\mathscr{V}_{H}$, multiscale spectral coarse space $\widetilde{\mathscr{V}_{H}}$, and multiscale coarse space $\mathscr{V}_{H}^{m s}$.

\begin{tabular}{|c|c|c|c|c|c|c|c|c|c|}
\cline { 2 - 10 } \multicolumn{1}{c|}{} & \multicolumn{4}{c|}{$\mathscr{V}_{H}$} & \multicolumn{3}{c|}{$\widetilde{\mathscr{V}}_{H}$} & \multicolumn{3}{c|}{$\mathscr{V}_{H}^{m s}$} \\
\hline$\frac{\kappa_{\max }}{\kappa_{\min }}$ & $\#$ iter. & $\operatorname{dim} \mathscr{V}_{H}$ & cond. \# & \# iter. & $\operatorname{dim} \widetilde{\mathscr{V}}_{H}$ & cond. \# & \# iter & $\operatorname{dim} \mathscr{V}_{H}^{m s}$ & cond \# \\
\hline $1 e 2$ & 22 & 163 & 12.15 & 21 & 44 & 10.81 & 19 & 49 & 8.70 \\
\hline $1 e 3$ & 18 & 612 & 8.42 & 20 & 60 & 9.86 & 35 & 49 & $5.97 \mathrm{e} 1$ \\
\hline $1 e 4$ & 15 & 838 & 4.92 & 22 & 60 & 10.90 & 44 & 49 & $5.63 \mathrm{e} 2$ \\
\hline $1 e 5$ & 16 & 838 & 4.92 & 22 & 60 & 11.01 & 53 & 49 & $5.59 \mathrm{e} 3$ \\
\hline $1 e 6$ & 17 & 838 & 4.92 & 22 & 60 & 11.01 & 66 & 49 & $5.59 \mathrm{e} 4$ \\
\hline
\end{tabular}

In Table 5 we present the results when the preconditioner is based on the spectral coarse space $\mathscr{V}_{H}$ (columns 2-4), the multiscale spectral coarse space $\widetilde{\mathscr{V}}_{H}$ (columns $5-7$ ), and the multiscale coarse space $\mathscr{V}_{H}^{m s}$ (columns 8-10). Comparing the data for the spaces $\mathscr{V}_{H}$ and $\widetilde{\mathscr{V}_{H}}$ shows that the number of PCG-iterations and the estimated condition number of the preconditioned system are robust with respect to the contrast $\kappa_{\max } / \kappa_{\min }$. We can also observe that when using the spectral coarse space $\mathscr{V}_{H}$ the dimension of the coarse space increases as the contrast increases $\kappa_{\max } / \kappa_{\min }$, which is in agreement with the analysis of Section 4.1. The decrease in the estimated condition number when going from $\kappa_{\max } / \kappa_{\min }=1 e 2$ to $\kappa_{\max } / \kappa_{\min }=1 e 3$ and further to $\kappa_{\max } / \kappa_{\min }=1 e 4$ can be explained by the fact that for higher contrasts more eigenvalues are below the prescribed threshold, yielding a higher dimensional coarse space and a lower condition number. However, it is important to note that the dimension of the coarse space reaches a maximum for $\kappa_{\max } / \kappa_{\min }$ above a certain threshold. As we can see for $\kappa_{\max } / \kappa_{\min }$ in the range $1 e 4, \ldots, 1 e 6$, the dimension of the coarse space stays the same. By the analysis in Section 4.1 we know that there is only a finite number of asymptotically small (with the contrast tending to infinity) generalized eigenvalues. The reported data can be seen as evidence that for this specific configuration we have reached this asymptotic regime for $\kappa_{\max } / \kappa_{\min }=1 e 4$.

In columns 8-10 we present the numerical results of the algorithm when the preconditioner is based on the multiscale coarse space $\mathscr{V}_{H}^{m s}$, which consist of one basis function per interior coarse node. As we can see from the data, the number of PCG-iterations as well as the condition number of the preconditioned system grow steadily with the growth of the contrast.

The important point to observe when using the multiscale spectral coarse space $\widetilde{\mathscr{V}}_{H}$ (see columns $5-7$ of Tab. 5) is that its dimension is drastically reduced compared to the spectral coarse space $\mathscr{V}_{H}$. In our specific example, the largest dimension of the multiscale spectral coarse space $\widetilde{\mathscr{V}_{H}}$, which is constructed using the multiscale partition of unity $\left\{\widetilde{\xi}_{j}\right\}_{j=1}^{n_{x}}$, is 60 , compared to the dimension 838 of the spectral coarse space $\mathscr{V}_{H}$, which is based on the standard partition of unity $\left\{\xi_{j}\right\}_{j=1}^{n_{x}}$. One is generally interested in keeping the dimension of the coarse space as small as possible, especially when the problem is solved multiple times. The data is a confirmation of our reasoning in Section 5.

We note that the dimension of the multiscale spectral coarse space is slightly larger than that of the standard coarse space (60 compared to 49 for the highest contrast); however, the cost of subdomain solves is the same in both cases and it is usually higher than the cost of solving the coarse problem.

\subsection{Numerical experiments for geometries 3 and 4}

In Table 6 we present the numerical results for the scalar elliptic equation of second order in Galerkin formulation from Section 4.1 for highly heterogeneous permeability distributions shown in Figure 5. Again, the boundary conditions and $f$ are chosen as in Section 6.2.1. Geometry 3 represents a rather challenging example: the permeability field is highly heterogeneous with more than 4000 small and about 100 large randomly distributed inclusions. We consider this a challenging test for the robustness of the iterative method by performing a relatively small number of iterations using a coarse space of low dimension. Here, we have used a $16 \times 16$ coarse 
TABLE 6. Scalar elliptic problem - Galerkin formulation: numerical results for permeability fields shown in Figure 5 using multiscale spectral coarse spaces $\widetilde{\mathscr{V}}_{H}$.

\begin{tabular}{|c|c|c|c|c|c|c|}
\cline { 2 - 7 } \multicolumn{1}{c|}{} & \multicolumn{3}{c|}{ Geometry 3} & \multicolumn{3}{c|}{ Geometry 4} \\
\hline$\kappa_{\text {max }} / \kappa_{\text {min }}$ & \# iter. & $\operatorname{dim} \widetilde{\mathscr{V}}_{H}$ & cond. \# & \# iter. & $\operatorname{dim} \widetilde{\mathscr{V}}_{H}$ & cond. \# \\
\hline $1 e 2$ & 22 & 209 & 11.2 & 19 & 217 & 8.47 \\
\hline $1 e 3$ & 24 & 259 & 15.2 & 20 & 221 & 9.48 \\
\hline $1 e 4$ & 23 & 275 & 11.2 & 22 & 244 & 11.5 \\
\hline $1 e 5$ & 24 & 277 & 11.2 & 25 & 317 & 16.3 \\
\hline $1 e 6$ & 27 & 293 & 11.7 & 23 & 397 & 11.7 \\
\hline
\end{tabular}

mesh and subdivided each coarse cell into $16 \times 16$ subcells to obtain a $256 \times 256$ fine mesh. The preconditioner is based on the multi-scale spectral coarse space $\widetilde{\mathscr{V}_{H}}$. The dimension of the fine space is 66049 while the dimension of the coarse space is at most 293. As we can see, the condition number of the preconditioned system is robust with respect to the contrast and the dimension of the coarse space is quite small.

Geometry 4 (see Fig. 5) represents a more challenging problem in that it is no longer a binary medium, i.e., $\kappa$ assumes many and not just two extreme values. The geometry is generated by setting $\kappa$ in a fine mesh cell to $10^{\gamma \text { rand }}$, where rand denotes a uniformly distributed random number and $\gamma=2, \ldots, 6$. This produces a random field $10^{\gamma \eta(\boldsymbol{x})}$ where $\eta(\boldsymbol{x})$ is a realization of a spatially uncorrelated random field. This yields a "background" on top of which we put randomly generated inclusions similar to geometry 3 . In Table 6 (columns $5-7$ ) we report the numerical results using the preconditioner based on the multiscale spectral space $\widetilde{\mathscr{V}}_{H}$. As we can see, the number of PCG iterations and the condition number of the preconditioned system are robust with respect to increases in the contrast. It is furthermore important to note that, even for this random case, the dimension of the coarse space stays reasonably small (at most 397) compared to the dimension of the fine space, i.e., 66049. This exemplifies the robustness and applicability of the numerical method developed above.

\section{Conclusions}

The theory developed above introduces a method for constructing stable decompositions with respect to symmetric positive definite operators. The robustness with respect to problem and mesh parameters is proved under rather general assumptions. We have furthermore applied this abstract framework to several important cases, i.e., the scalar elliptic equation in Galerkin and mixed formulation, Stokes' equations, and Brinkman's equations. For the scalar elliptic equation in Galerkin formulation, we have additionally presented a strategy of reducing the dimension of the coarse space in the stable decomposition. To verify our analytical results, we have performed several numerical experiments, which are in coherence with our theory and show the usefulness of the method.

Acknowledgements. The research of Y. Efendiev, J. Galvis and R. Lazarov was supported in parts by award KUS-C1016-04, made by King Abdullah University of Science and Technology (KAUST). The research of R. Lazarov and J. Willems was supported in parts by NSF Grant DMS-1016525.

\section{REFERENCES}

[1] R.A. Adams, Sobolev Spaces, 1st edition. Pure Appl. Math. Academic Press, Inc. (1978).

[2] W. Bangerth, R. Hartmann and G. Kanschat, deal.II - a general purpose object oriented finite element library. ACM Trans. Math. Softw. 33 (2007) 24/1-24/27. 
[3] J.H. Bramble, Multigrid Methods, 1st edition. Longman Scientific \& Technical, Essex (1993).

[4] S.C. Brenner and L.R. Scott, The Mathematical Theory of Finite Element Methods, 2nd edition. Springer (2002).

[5] H.C. Brinkman, A calculation of the viscouse force exerted by a flowing fluid on a dense swarm of particles. Appl. Sci. Res. A1 (1947) 27-34.

[6] T. Chartier, R.D. Falgout, V.E. Henson, J. Jones, T. Manteuffel, S. McCormick, J. Ruge and P.S. Vassilevski, Spectral AMGe ( $\rho \mathrm{AMGe}$. SIAM J. Sci. Comput. 25 (2003) 1-26.

[7] M. Dryja, M.V. Sarkis and O.B. Widlund, Multilevel Schwarz methods for elliptic problems with discontinuous coefficients in three dimensions. Numer. Math. 72 (1996) 313-348.

[8] Y. Efendiev and T.Y. Hou, Multiscale finite element methods, Theory and applications. Surveys and Tutorials in Appl. Math. Sci. Springer, New York 4 (2009).

[9] R.E. Ewing, O. Iliev, R.D. Lazarov, I. Rybak and J. Willems, A simplified method for upscaling composite materials with high contrast of the conductivity. SIAM J. Sci. Comput. 31 (2009) 2568-2586.

[10] J. Galvis and Y. Efendiev, Domain decomposition preconditioners for multiscale flows in high-contrast media. Multiscale Model. Simul. 8 (2010) 1461-1483.

[11] J. Galvis and Y. Efendiev, Domain decomposition preconditioners for multiscale flows in high contrast media: reduced dimension coarse spaces. Multiscale Model. Simul. 8 (2010) 1621-1644.

[12] V. Girault and P.-A. Raviart, Finite element methods for Navier-Stokes equations, Springer Series in Comput. Math. Theory and Algorithms 5 (1986).

[13] I.G. Graham, P.O. Lechner and R. Scheichl, Domain decomposition for multiscale PDEs. Numer. Math. 106 (2007) 589-626.

[14] P. Grisvard, Elliptic problems in nonsmooth domains, Monographs and Studies in Mathematics. Pitman Advanced Publishing Program, Boston, MA 24 (1985).

[15] W. Hackbusch, Multi-Grid Methods and Applications, 2nd edition. Springer Series in Comput. Math. Springer, Berlin (2003).

[16] T.Y. Hou, X.-H. Wu and Z. Cai, Convergence of a multiscale finite element method for elliptic problems with rapidly oscillating coefficients. Math. Comp. 68 (1999) 913-943.

[17] A. Klawonn, O.B. Widlund and M. Dryja, Dual-primal FETI methods for three-dimensional elliptic problems with heterogeneous coefficients. SIAM J. Numer. Anal. 40 (2002) 159-179 (electronic).

[18] J. Mandel and M. Brezina, Balancing domain decomposition for problems with large jumps in coefficients. Math. Comp. 65 (1996) 1387-1401.

[19] T.P.A. Mathew, Domain Decomposition Methods for the Numerical Solution of Partial Differential Equations. Lect. Notes Comput. Sci. Eng. Springer, Berlin Heidelberg (2008).

[20] S.V. Nepomnyaschikh, Mesh theorems on traces, normalizations of function traces and their inversion. Sov. J. Numer. Anal. Math. Modelling 6 (1991) 151-168.

[21] C. Pechstein and R. Scheichl, Analysis of FETI methods for multiscale PDEs. Numer. Math. 111 (2008) $293-333$.

[22] C. Pechstein and R. Scheichl, Analysis of FETI methods for multiscale PDEs - Part II: interface variation. To appear in Numer. Math.

[23] M. Reed and B. Simon, Methods of Modern Mathematical Physics IV: Analysis of Operators. Academic Press, New York (1978).

[24] M.V. Sarkis, Schwarz Preconditioners for Elliptic Problems with Discontinuous Coefficients Using Conforming and NonConforming Elements. Ph.D. thesis, Courant Institute, New York University (1994).

[25] M.V. Sarkis, Nonstandard coarse spaces and Schwarz methods for elliptic problems with discontinuous coefficients using non-conforming elements. Numer. Math. 77 (1997) 383-406.

[26] B.F. Smith, P.E. Bjørstad and W.D. Gropp, Domain Decomposition, Parallel Multilevel Methods for Elliptic Partial Differential Equations, 1st edition. Cambridge University Press, Cambridge (1996).

[27] A. Toselli and O. Widlund, Domain Decomposition Methods - Algorithms and Theory. Springer Series in Comput. Math. (2005).

[28] J. Van Lent, R. Scheichl and I.G. Graham, Energy-minimizing coarse spaces for two-level Schwarz methods for multiscale PDEs. Numer. Linear Algebra Appl. 16 (2009) 775-799.

[29] P.S. Vassilevski, Multilevel block-factrorization preconditioners. Matrix-based analysis and algorithms for solving finite element equations. Springer-Verlag, New York (2008).

[30] J. Wang and X. Ye, New finite element methods in computational fluid dynamics by H(div) elements. SIAM J. Numer. Anal. 45 (2007) 1269-1286.

[31] J. Willems, Numerical Upscaling for Multiscale Flow Problems. Ph.D. thesis, University of Kaiserslautern (2009).

[32] J. Xu and L.T. Zikatanov, On an energy minimizing basis for algebraic multigrid methods. Comput. Visualisation Sci. 7 (2004) 121-127. 